\title{
Distributed Solution of Optimal Control Problems Governed by Parabolic Equations *
}

\author{
Matthias Heinkenschloss ${ }^{\dagger} \quad$ Michael Herty ${ }^{\ddagger}$ \\ December 23, 2004 \\ (revised February 11, 2005)
}

\begin{abstract}
We present a spatial domain decomposition (DD) method for the solution of discretized parabolic linear-quadratic optimal control problems. Our DD preconditioners are extensions of Neumann-Neumann DD methods, which have been successfully applied to the solution of single elliptic partial differential equations and of linear-quadratic optimal control problems governed by elliptic equations.

We use a decomposition of the spatial domain into non-overlapping subdomains. The optimality conditions for the parabolic linear-quadratic optimal control problem is split into smaller problems restricted to spatial subdomain-time cylinders. These subproblems correspond to parabolic linear-quadratic optimal control problems on subdomains with Dirichlet data on interfaces. The coupling of these subdomain problems leads to a Schur complement system in which the unknowns are the state and adjoint variables on the subdomain interfaces in space and time.

The Schur complement system is solved using a preconditioned Krylov subspace method. The preconditioner is obtained from the solution of appropriate subdomain parabolic linearquadratic optimal control problems. The dependence of the performance of these preconditioners on mesh size and subdomain size is studied numerically. Our tests indicate that their dependence on mesh size and subdomain size is similar to that of its counterpart applied to elliptic equations only. Our tests also suggest that the preconditioners are insensitive to the size of the control regularization parameter.
\end{abstract}

Key words Optimal control, parabolic equations, domain decomposition, NeumannNeumann methods

AMS subject classifications 49M05, 65N55, 65F10, 90C06, 90C20

${ }^{*}$ This work was supported in part by NSF Grants ACI-0121360 and CNS-0435425 and DAAD Grant D/04/23833.

${ }^{\dagger}$ Department of Computational and Applied Mathematics, MS-134, Rice University, 6100 Main Street, Houston, TX 77005-1892. E-mail: heinken@rice.edu

${ }^{\ddagger}$ Fachbereich Mathematik, Technische Universität Kaiserslautern, Postfach 30 49, D-67653 Kaiserslautern, Germany. E-mail: herty@mathematik.uni-kl.de 


\section{Introduction}

This paper presents a spatial domain decomposition method for the solution of linear-quadratic parabolic optimal control problems. Such problems arise directly in many applications, but also as subproblems in Newton or sequential quadratic programming methods for the solution of nonlinear parabolic optimal control problems. The motivation for this work is threefold. First, our approach attempts to address the storage issue that arises in the numerical solution of parabolic optimal control problems out of the strong coupling in space and time of state (PDE solution), the adjoint, and the control. Secondly, our spatial domain decomposition method introduces parallelism at the optimization level. The last motivation arises from the availability of sensor networks that offer in-network computing capabilities, allow neighbor-to-neighbor communication, but for which global communication requires large amounts of resources because of communication bandwidth and battery power limitations. Our domain decomposition method offers the possibility for in-network computing, in which the global problem is solved using spatially distributed processors that communicate with their neighbors.

Domain decomposition methods have been applied previously to linear-quadratic time dependent optimal control problems. They split into time domain decomposition methods $[4,10,13,14$, 17] and spatial domain decomposition methods [1, 2, 3]. Like [1, 2, 3], the approach in this paper is also based on a decomposition of the spatial domain. The resulting subproblems are smaller linear-quadratic parabolic optimal control problems posed on a spatial subdomain-time cylinder. The difference between the approaches $[1,2,3]$ and our approach lies in the way the subdomain problems are coupled and in the solution method for the coupled subdomain problems.

Our spatial domain decomposition method for linear-quadratic parabolic optimal control problems is based on the so-called Neumann-Neumann domain decomposition methods. Of the domain decomposition method for elliptic partial differential equations (PDEs), Neumann-Neumann methods are among the most successful ones. Their derivation and discussions of their convergence properties can be found in the books $[18,20,21]$ and the references given therein. Recently, Neumann-Neumann methods were generalized to solve linear-quadratic elliptic optimal control problems. The results in $[11,12]$ have shown that their performance on linear-quadratic elliptic optimal control model problems is comparable to their good performance for single elliptic PDEs. This paper extends Neumann-Neumann methods to the solution of linear-quadratic parabolic optimal control problems.

To illustrate our ideas, we consider the example problem

$$
\begin{array}{rlr}
\operatorname{minimize} & \frac{\alpha_{1}}{2} \int_{0}^{T} \int_{\Omega}(y(x, t)-\hat{y}(x, t))^{2} d x d t+\frac{\alpha_{2}}{2} \int_{\Omega}\left(y(x, T)-\hat{y}_{T}(x)\right)^{2} d x \\
& +\frac{\alpha_{3}}{2} \int_{0}^{T} \int_{\Omega} u^{2}(x, t) d x d t, & \\
\text { subject to } & \partial_{t} y(x, t)-\Delta y(x, t)=f(x, t)+u(x, t) & \text { in } \Omega \times(0, T), \\
y(x, t)=0 & \text { on } \partial \Omega \times(0, T), \\
y(x, 0)=y_{0}(x) & \text { in } \Omega,
\end{array}
$$


where $\hat{y}, \hat{y}_{T}, f$ are given functions and $\alpha_{1}, \alpha_{2} \geq 0, \alpha_{3}>0$ are given parameters. The problem (1.1) has to be solved for $y$ and $u$. Detailed model problem assumptions will be introduced in the next section.

Section 3 introduces the domain decomposition formulation for a discretization of (1.1). We decompose $\Omega$ into nonoverlapping domains $\Omega_{i}, i=1, \ldots, s$. Essentially, we decompose the system of optimality conditions for (1.1) by expressing states, controls, and adjoints inside the subdomains $\Omega_{i} \times(0, T), i=1, \ldots, s$, as functions of the states and adjoints on the subdomain interfaces $\left(\partial \Omega_{i} \backslash \partial \Omega\right) \times(0, T)$. Transmission conditions that couple the subdomain problems are then viewed as operator equations in states and adjoints restricted to the subdomain interfaces $\left(\partial \Omega_{i} \backslash \partial \Omega\right) \times(0, T)$. As we have mentioned before, our domain decomposition method extends Neumann-Neumann domain decomposition methods well known for the solution of elliptic PDEs to the solution of linear-quadratic parabolic optimal control problems. Often the discretization of the system of optimality conditions for (1.1) is not equal to the system of optimality conditions for the discretization of (1.1). Therefore, we formulate our domain decomposition method not for the infinite dimensional problem, but for a discretization of (1.1). This ensures that the problem solved by our domain decomposition method is the discretization of (1.1), i.e. the problem one would typically solve and not some perturbation of it.

Section 4 reports on some initial numerical results.

\section{The Example Problem}

In this section, we define the setting for the model problem (1.1), recall a result on the existence and uniqueness of its solution, and review the well-known necessary and sufficient optimality conditions. Furthermore, we introduce a discretization of (1.1). The results in this section are well known, but are recalled here to serve as the background for our domain decomposition formulation.

\subsection{The Infinite Dimensional Problem}

Let $\Omega \subset \mathbb{R}^{d}, d=1,2,3$, be an open, bounded set with Lipschitz boundary (if $d=2$ or 3 ). We consider the state space

$$
\mathcal{Y}=W(0, T)=\left\{y: y \in L^{2}\left(0, T ; H_{0}^{1}(\Omega)\right), y^{\prime} \in L^{2}\left(0, T ; H^{-1}(\Omega)\right)\right\}
$$

and the control space

$$
\mathcal{U}=L^{2}\left(0, T ; L^{2}(\Omega)\right) .
$$

We assume that $y_{0}, \hat{y}_{T} \in L^{2}(\Omega), \hat{y} \in L^{2}\left(0, T ; L^{2}(\Omega)\right), f \in L^{2}\left(0, T ; L^{2}(\Omega)\right)$ are given functions, and that $\alpha_{1}, \alpha_{2} \geq 0, \alpha_{3}>0$ are given parameters. We define the bilinear forms

$$
\begin{array}{ll}
a: H_{0}^{1}(\Omega) \times H_{0}^{1}(\Omega) \rightarrow \mathbb{R}, & a(y, \psi)=\int_{\Omega} \nabla y(x) \nabla \psi(x) d x, \\
b: L^{2}(\Omega) \times H_{0}^{1}(\Omega) \rightarrow \mathbb{R}, & b(u, \psi)=-\int_{\Omega} u(x) \psi(x) d x
\end{array}
$$


and we use $\langle\cdot, \cdot\rangle_{L^{2}(\Omega)}$ and $\|\cdot\|_{L^{2}(\Omega)}$ to denote the inner product and the norm in $L^{2}(\Omega)$.

We are interested in the solution $y \in Y, u \in U$ of the optimal control problem

$$
\begin{array}{ll}
\operatorname{minimize} & \frac{\alpha_{1}}{2} \int_{0}^{T}\|y(t)-\hat{y}(t)\|_{L^{2}(\Omega)}^{2} d t+\frac{\alpha_{2}}{2}\left\|y(T)-\hat{y}_{T}\right\|_{L^{2}(\Omega)}^{2} \\
& +\frac{\alpha_{3}}{2} \int_{0}^{T}\|u(t)\|_{L^{2}(\Omega)}^{2} d t \\
\text { subject to } & \left\langle y^{\prime}(t), \phi\right\rangle_{L^{2}(\Omega)}+a(y(t), \phi)+b(u(t), \phi) \\
& =\langle f(t), \phi\rangle_{L^{2}(\Omega)} \quad \forall \phi \in H_{0}^{1}(\Omega), \\
& y(0)=y_{0} .
\end{array}
$$

Theorem 2.1 The optimal control problem (2.1) has a unique solution $\left(u_{*}, y_{*}\right) \in \mathcal{U} \times \mathcal{Y}$, which, together with the adjoint variable $p_{*} \in \mathcal{Y}$, is characterized by the necessary and sufficient optimality conditions

$$
\begin{aligned}
-\left\langle p^{\prime}(t), \psi\right\rangle_{L^{2}(\Omega)}+a(\psi, p(t)) & =-\alpha_{1}\langle y(t)-\hat{y}(t), \psi\rangle_{L^{2}(\Omega)}, \\
p(T) & =-\alpha_{2}\left(y(T)-\hat{y}_{T}\right), \\
\alpha_{3}\langle u(t), \mu\rangle_{L^{2}(\Omega)}+b(\mu, p(t)) & =0, \\
\left\langle y^{\prime}(t), \phi\right\rangle_{L^{2}(\Omega)}+a(y(t), \phi)+b(u(t), \phi) & =\langle f(t), \phi\rangle_{L^{2}(\Omega)}, \\
y(0) & =y_{0} .
\end{aligned}
$$

for all $\psi, \phi \in H_{0}^{1}(\Omega), \mu \in L^{2}(\Omega)$.

Proof: The assertion of the theorem is well known and a proof can be found, e.g., in [16, p. 114,116].

\subsection{Discretization}

Let $V^{h} \subset H_{0}^{1}(\Omega), U^{h} \subset L^{2}(\Omega)$ be finite dimensional subspaces with bases $\psi_{1}, \ldots, \psi_{m}$ and $\mu_{1}, \ldots, \mu_{n}$, respectively. We approximate the states and controls by $y_{h} \in H^{1}\left(0, T ; V^{h}\right), u_{h} \in$ $L^{2}\left(0, T ; U^{h}\right)$ defined as

$$
y_{h}(t)=\sum_{l=1}^{m} y_{l}(t) \phi_{l}, \quad u_{h}(t)=\sum_{l=1}^{n} u_{l}(t) \mu_{l} .
$$

We define $\mathbf{A} \in \mathbb{R}^{m \times m}, \mathbf{B} \in \mathbb{R}^{m \times n}, \mathbf{M} \in \mathbb{R}^{m \times m}, \mathbf{Q} \in \mathbb{R}^{n \times n}, \mathbf{f} \in L^{2}\left(0, T ; \mathbb{R}^{m}\right), \mathbf{c} \in$ $L^{2}\left(0, T ; \mathbb{R}^{m}\right)$ and $\mathbf{d} \in \mathbb{R}^{m}$ as follows:

$$
\begin{gathered}
\mathbf{A}_{j l}=a\left(\phi_{l}, \phi_{j}\right), \quad \mathbf{M}_{j l}=\left\langle\phi_{l}, \phi_{j}\right\rangle_{L^{2}(\Omega)}, \\
\mathbf{c}_{j}(t)=-\alpha_{1}\left\langle\hat{y}(t), \phi_{j}\right\rangle_{L^{2}(\Omega)}, \quad \mathbf{f}_{j}(t)=\left\langle f(t), \phi_{j}\right\rangle_{L^{2}(\Omega)}, \quad \mathbf{d}_{j}=-\alpha_{2}\left\langle\hat{y}_{T}, \phi_{j}\right\rangle_{L^{2}(\Omega)}
\end{gathered}
$$


for $j, l=1, \ldots, m$, and

$$
\mathbf{B}_{j l}=b\left(\mu_{l}, \phi_{j}\right), \quad \mathbf{Q}_{j l}=\left\langle\mu_{l}, \mu_{j}\right\rangle_{L^{2}(\Omega)}
$$

for $j, l=1, \ldots, n$. We set $\mathbf{y}(t)=\left(y_{1}(t), \ldots, y_{m}(t)\right)^{T}$ and $\mathbf{u}(t)=\left(u_{1}(t), \ldots, u_{n}(t)\right)^{T}$ where $y_{i}, u_{i}, p_{i}$ are the functions in (2.3).

We now replace $y, u$ by $y_{h}, u_{h}$ defined in (2.3) and require (2.1b) to hold for $\phi=\phi_{l}, l=$ $1, \ldots, m$. This finite element semi-discretization of the optimal control problem (2.1) leads to a large-scale linear-quadratic problem of the form

$$
\begin{aligned}
& \text { minimize } \int_{0}^{T} \frac{\alpha_{1}}{2} \mathbf{y}(t)^{T} \mathbf{M y}(t)+\mathbf{c}(t)^{T} \mathbf{y}(t) d t+\frac{\alpha_{2}}{2} \mathbf{y}(T)^{T} \mathbf{M y}(T)+\mathbf{d}^{T} \mathbf{y}(T) \\
& +\int_{0}^{T} \frac{\alpha_{3}}{2} \mathbf{u}(t)^{T} \mathbf{Q u}(t) d t \\
& \text { subject to } \mathbf{M y}^{\prime}(t)+\mathbf{A y}(t)+\mathbf{B u}(t)=\mathbf{f}(t), \quad t \in(0, T) \\
& \mathbf{y}(0)=\mathbf{y}_{0}
\end{aligned}
$$

To fully discretize (2.4), we use the backward Euler method on an equidistant time grid

$$
t_{k}=k \Delta t, \quad k=0, \ldots, K
$$

with time step size $\Delta t=T / K$. We use $\mathbf{y}_{k}$ and $\mathbf{u}_{k}$ to denote the approximate semi-discretized state $\mathbf{y}$ and control $\mathbf{u}$ at $t_{k}$. Our discretization of (2.4) is given by

$$
\begin{aligned}
& \operatorname{minimize} \sum_{k=1}^{K} \frac{\alpha_{1} \Delta t}{2} \mathbf{y}_{k}^{T} \mathbf{M} \mathbf{y}_{k}+\Delta t \mathbf{c}\left(t_{k}\right)^{T} \mathbf{y}_{k}+\frac{\alpha_{2}}{2} \mathbf{y}_{K}^{T} \mathbf{M} \mathbf{y}_{K}+\mathbf{d}^{T} \mathbf{y}_{K} \\
&+\sum_{k=1}^{K} \frac{\alpha_{3} \Delta t}{2} \mathbf{u}_{k}^{T} \mathbf{Q} \mathbf{u}_{k}, \\
& \text { subject to } \mathbf{M} \frac{\mathbf{y}_{k+1}-\mathbf{y}_{k}}{\Delta t}+\mathbf{A} \mathbf{y}_{k+1}+\mathbf{B} \mathbf{u}_{k+1}=\mathbf{f}\left(t_{k+1}\right), \quad k=0, \ldots, K-1 .
\end{aligned}
$$

In (2.5), $\mathbf{y}_{0}$ is the given vector of initial data.

Theorem 2.2 The vectors $\mathbf{y}_{1}, \ldots, \mathbf{y}_{K}, \mathbf{u}_{1}, \ldots, \mathbf{u}_{K}$ solve (2.5) if and only if there exist $\mathbf{p}_{1}, \ldots, \mathbf{p}_{K}$ such that

$$
\begin{array}{rlrl}
-\mathbf{M} \frac{\mathbf{p}_{k+1}-\mathbf{p}_{k}}{\Delta t}+\mathbf{A}^{T} \mathbf{p}_{k}+\alpha_{1} \mathbf{M} \mathbf{y}_{k} & =-\mathbf{c}\left(t_{k}\right), & k=1, \ldots, K-1, \\
\left(\frac{1}{\Delta t} \mathbf{M}+\mathbf{A}^{T}\right) \mathbf{p}_{K}+\left(\alpha_{1}+\frac{\alpha_{2}}{\Delta t}\right) \mathbf{M} \mathbf{y}_{K} & =-\mathbf{c}\left(t_{K}\right)-\frac{1}{\Delta t} \mathbf{d}, & \\
\alpha_{3} \mathbf{Q} \mathbf{u}_{k}+\mathbf{B}^{T} \mathbf{p}_{k}=0, & k=1, \ldots, K, \\
\mathbf{M} \frac{\mathbf{y}_{k}-\mathbf{y}_{k-1}}{\Delta t}+\mathbf{A y}_{k}+\mathbf{B} \mathbf{u}_{k}=\mathbf{f}\left(t_{k}\right), & k=1, \ldots, K .
\end{array}
$$


Proof: The Lagrangian for (2.5) is given by

$$
\begin{aligned}
& L\left(\mathbf{y}_{1}, \ldots, \mathbf{y}_{K}, \mathbf{u}_{1}, \ldots, \mathbf{u}_{K}, \mathbf{p}_{1}, \ldots, \mathbf{p}_{K}\right) \\
& =\sum_{k=1}^{K} \frac{\alpha_{1} \Delta t}{2} \mathbf{y}_{k}^{T} \mathbf{M} \mathbf{y}_{k}+\Delta t \mathbf{c}\left(t_{k}\right)^{T} \mathbf{y}_{k}+\frac{\alpha_{2}}{2} \mathbf{y}_{K}^{T} \mathbf{M} \mathbf{y}_{K}+\mathbf{d}^{T} \mathbf{y}_{K}+\sum_{k=1}^{K} \frac{\alpha_{3} \Delta t}{2} \mathbf{u}_{k}^{T} \mathbf{Q} \mathbf{u}_{k}, \\
& \quad+\sum_{k=0}^{K-1} \mathbf{p}_{k+1}^{T} \Delta t\left(\mathbf{M} \frac{\mathbf{y}_{k+1}-\mathbf{y}_{k}}{\Delta t}+\mathbf{A} \mathbf{y}_{k+1}+\mathbf{B} \mathbf{u}_{k+1}-\mathbf{f}\left(t_{k+1}\right)\right) .
\end{aligned}
$$

In the definition of the Lagrangian we use the weighted Euclidean inner product between the Lagrange multipliers $\mathbf{p}_{k+1}$ and the constraints, with weight given by $\Delta t$, since it corresponds to an integral for the semi-discrete problem (2.4). Of course, this weighting is equivalent to a scaling of the Lagrange multipliers.

The problem (2.5) is convex, linear-quadratic. The necessary and sufficient optimality conditions for (2.5) are obtained by setting the derivatives of the Lagrangian to zero, which gives (2.6).

\section{Domain Decomposition Formulation of the Example Prob- lem}

In this section we introduce our spatial domain decomposition algorithm for the fully discretized problem. This study is useful because the discretization of the adjoint equations (2.2a) is usually not equal to the adjoint equations of the discretization of the optimal control problem (2.5). In this section we apply the spatial domain decomposition algorithm to the optimality conditions for the discretization of the optimal control problem (2.5). Hence, the solution generated by our domain decomposition algorithm is the solution of the full discretization of (2.1).

\subsection{Domain Decomposition in Space}

We discretize (2.1) using conforming linear finite elements. Thus, given a triangulation $\left\{T_{l}\right\}$ of $\Omega$, the space $V^{h}$ used in the discretization of the states is given by

$$
V^{h}=\left\{v \in H_{0}^{1}(\Omega):\left.v\right|_{T_{l}} \in P^{1}\left(T_{l}\right) \text { for all } l\right\}
$$

We divide $\Omega$ into nonoverlapping subdomains $\Omega_{i}, i=1, \ldots, s$, such that each $T_{l}$ belongs to exactly one $\bar{\Omega}_{i}$. We define $\Gamma_{i}=\partial \Omega_{i} \backslash \partial \Omega$ and $\Gamma=\cup_{i=1}^{s} \Gamma_{i}$.

Let $\left\{x_{j}\right\}_{j=1}^{m}$ be the set of vertices of $\left\{T_{l}\right\}$ that lie inside $\Omega_{i}$ and let $\left\{\phi_{j}\right\}_{j=1}^{m}$ be the piecewise linear nodal basis for $V^{h}$. Let $m_{I}^{i}$ be the number of vertices in $\Omega_{i}$, let $m_{\Gamma}^{i}$ be the number of vertices on $\Gamma \cap \partial \Omega_{i}$, and let $m_{\Gamma}=\sum_{i=1}^{s} m_{\Gamma}^{i}$ be the number of vertices on the subdomain interfaces $\Gamma$. Hence the number of discretized state variables for a given time $t$ is given by $m=m_{\Gamma}+\sum_{i=1}^{s} m_{I}^{i}$.

For the semidiscretization of the control, we use functions that are continuous on each $\Omega_{i}, i=$ $1, \ldots, s$, and linear on each $\Omega_{i} \cap T_{l}$, but that are not assumed to be continuous at $\partial \Omega_{i} \cap \partial \Omega_{j}, i \neq j$. In 
particular, for each point $x_{l} \in \partial \Omega_{i} \cap \partial \Omega_{j}, i \neq j$, there are two discrete controls $u_{h}\left(x_{k_{i}}, t\right), u_{h}\left(x_{k_{j}}, t\right)$ belonging to subdomains $\Omega_{i}$ and $\Omega_{j}$, respectively (see the right plot in Figure 3.1). Hence, our control discretization depends on the partition $\left\{\Omega_{i}\right\}_{i=1}^{d}$ of the domain $\Omega$. See $[11,12]$ for more discussion. We define the discrete spaces

$$
U_{i}^{h}=\left\{u \in C^{0}\left(\Omega_{i}\right): u \text { is linear on } \Omega_{i} \cap T_{l} \text { for all } T_{l} \subset \bar{\Omega}_{i}\right\} .
$$

We identify $U_{i}^{h}$ with a subspace of $L^{2}(\Omega)$ by extending functions $u_{i} \in U_{i}^{h}$ by zero onto $\Omega$. We define

$$
U^{h}=\cup_{i=1}^{s} U_{i}^{h} \subset L^{2}(\Omega)
$$
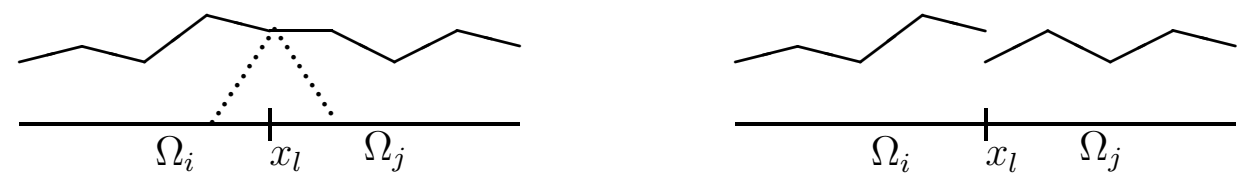

Figure 3.1: Sketch of the Control Discretization in Space for the Case $\Omega \subset \mathbb{R}$

Let $\left\{\mu_{j}^{i}\right\}_{j=1}^{n^{i}}$ be the piecewise linear nodal basis for $U_{i}^{h}$, where $n^{i}$ is the number of vertices in $\overline{\Omega_{i}}$. We identify $\mu_{j}^{i}$ with a function in $L^{2}(\Omega)$ by extending $\mu_{j}^{i}$ by zero outside $\overline{\Omega_{i}}$. We set

$$
\mu_{1}=\mu_{1}^{1}, \ldots, \mu_{n^{i}}=\mu_{n^{1}}^{1}, \mu_{n^{1}+1}=\mu_{1}^{2}, \ldots, \mu_{n^{1}+n^{2}}=\mu_{n^{2}}^{2}, \ldots
$$

The number of discretized control variables for a given time $t$ is given by $n=\sum_{i=1}^{s} n^{i}$.

\subsection{Decomposition of the Discretized Example Problem}

We can use the decomposition of $\Omega$ to decompose the matrices $\mathbf{A}$, etc. For $i=1, \ldots, s$ we define

$$
\begin{array}{ll}
a_{i}: H^{1}\left(\Omega_{i}\right) \times H^{1}\left(\Omega_{i}\right) \rightarrow \mathbb{R}, & a_{i}\left(y_{i}, \psi_{i}\right)=\int_{\Omega_{i}} \nabla y_{i}(x) \nabla \psi_{i}(x) d x, \\
b_{i}: L^{2}\left(\Omega_{i}\right) \times H^{1}\left(\Omega_{i}\right) \rightarrow \mathbb{R}, & b_{i}(u, \psi)=-\int_{\Omega_{i}} u_{i}(x) \psi_{i}(x) d x .
\end{array}
$$

For $i=1, \ldots, s$, we define the submatrices

$$
\begin{array}{ll}
\left(\mathbf{A}_{I I}^{i}\right)_{j l}=a_{i}\left(\phi_{l}, \phi_{j}\right), & x_{j}, x_{l} \in \Omega_{i}, \\
\left(\mathbf{A}_{I \Gamma}^{i}\right)_{j l}=a_{i}\left(\phi_{l}, \phi_{j}\right), & x_{j} \in \Omega_{i}, x_{l} \in \partial \Omega_{i} \backslash \partial \Omega, \\
\left(\mathbf{A}_{\Gamma I}^{i}\right)_{j l}=a_{i}\left(\phi_{l}, \phi_{j}\right), & x_{j} \in \partial \Omega_{i} \backslash \partial \Omega, x_{l} \in \Omega_{i}, \\
\left(\mathbf{A}_{\Gamma \Gamma}^{i}\right)_{j l}=a_{i}\left(\phi_{l}, \phi_{j}\right), & x_{j}, x_{l} \in \partial \Omega_{i} \backslash \partial \Omega,
\end{array}
$$

and $\mathbf{A}_{\Gamma \Gamma}=\sum_{i=1}^{s} \mathbf{A}_{\Gamma \Gamma}^{i}$. After a suitable reordering of rows and columns, the stiffness matrix can be written as

$$
\mathbf{A}=\left(\begin{array}{cccc}
\mathbf{A}_{I I}^{1} & & & \mathbf{A}_{I \Gamma}^{1} \\
& \ddots & & \vdots \\
& & \mathbf{A}_{I I}^{s} & \mathbf{A}_{I \Gamma}^{s} \\
\mathbf{A}_{\Gamma I}^{1} & \cdots & \mathbf{A}_{\Gamma I}^{s} & \mathbf{A}_{\Gamma \Gamma}
\end{array}\right) .
$$


A similar decompositions can be introduced for $\mathbf{M}$ and for the vectors $\mathbf{c}\left(t_{k}\right), \mathbf{f}\left(t_{k}\right), \mathbf{d}$. For example, for $i=1, \ldots, s$, we define

$$
\begin{array}{ll}
\left(\mathbf{d}_{I}^{i}\right)_{j}=-\alpha_{2}\left\langle\hat{y}_{T}, \phi_{j}\right\rangle_{L^{2}\left(\Omega_{i}\right)}, & x_{j} \in \Omega_{i}, \\
\left(\mathbf{d}_{\Gamma}^{i}\right)_{j}=-\alpha_{2}\left\langle\hat{y}_{T}, \phi_{j}\right\rangle_{L^{2}\left(\Omega_{i}\right.}, & x_{j} \in \partial \Omega_{i} \backslash \partial \Omega,
\end{array}
$$

and $\mathbf{d}_{\Gamma}=\sum_{i=1}^{s} \mathbf{d}_{\Gamma}^{i}$. After a suitable reordering, the vector $\mathbf{d}$ can be written as

$$
\mathbf{d}=\left(\begin{array}{c}
\mathbf{d}_{I}^{1} \\
\vdots \\
\mathbf{d}_{I}^{s} \\
\mathbf{d}_{\Gamma}
\end{array}\right)
$$

The vectors $\mathbf{y}_{k}, \mathbf{p}_{k}$ can be partitioned accordingly. For example, $\left(\mathbf{y}_{I}^{i}\right)_{k}$ denotes the subvector of $\mathbf{y}_{k}$ with indices $l$ such that $x_{l} \in \Omega_{i},\left(\mathbf{y}_{\Gamma}\right)_{k}$ denotes the subvector of $\mathbf{y}_{k}$ with indices $l$ such that $x_{l} \in \Gamma$, and $\left(\mathbf{y}_{\Gamma}^{i}\right)_{k}$ denotes the subvector of $\mathbf{y}_{k}$ with indices $l$ such that $x_{l} \in \Gamma \cap \partial \Omega_{i}$.

For $i=1, \ldots, s$, we define the submatrices

$$
\begin{aligned}
\left(\mathbf{B}_{I I}^{i}\right)_{j l} & =b_{i}\left(\mu_{l}, \phi_{j}\right), \quad x_{j} \in \Omega_{i}, x_{l} \in \overline{\Omega_{i}}, \\
\left(\mathbf{B}_{\Gamma I}^{i}\right)_{j l} & =b_{i}\left(\mu_{l}, \phi_{j}\right), \quad x_{j} \in \partial \Omega_{i} \backslash \partial \Omega, x_{l} \in \overline{\Omega_{i}}, \\
\left(\mathbf{Q}_{I I}^{i}\right)_{j l} & =\left\langle\mu_{l}, \phi_{j}\right\rangle_{L^{2}\left(\Omega_{i}\right)} \quad x_{j} \in \Omega_{i}, x_{l} \in \overline{\Omega_{i}} .
\end{aligned}
$$

After a suitable reordering of rows and columns, the matrix B can be written as

$$
\mathbf{B}=\left(\begin{array}{ccc}
\mathbf{B}_{I I}^{1} & & \\
& \ddots & \\
& & \mathbf{B}_{I I}^{s} \\
\mathbf{B}_{\Gamma I}^{1} & \cdots & \mathbf{B}_{\Gamma I}^{s}
\end{array}\right), \quad \mathbf{Q}=\left(\begin{array}{ccc}
\mathbf{Q}_{I I}^{1} & & \\
& \ddots & \\
& & \mathbf{Q}_{I I}^{s}
\end{array}\right) .
$$

Note that in our particular control discretization, all basis functions $\mu_{l}^{i}$ for the discretized control $u_{h}$ have support in only one subdomain $\Omega_{i}$ (see the right plot in Figure 3.1). Consequently, there is no $\mathbf{B}_{\Gamma \Gamma}^{i}$. The vectors $\mathbf{u}_{k}$ can be partitioned into

$$
\mathbf{u}_{k}=\left(\begin{array}{c}
\left(\mathbf{u}_{I}^{1}\right)_{k} \\
\vdots \\
\left(\mathbf{u}_{I}^{s}\right)_{k}
\end{array}\right)
$$

Due to our control discretization in space, there is no subvector $\left(\mathbf{u}_{\Gamma}\right)_{k}$ corresponding to interface nodes.

Let

$$
I_{\Gamma}^{i} \in \mathbb{R}^{m_{\Gamma}^{i} \times m_{\Gamma}}
$$

be the matrix with zero or one entries that extracts out of a vector $\mathbf{v}_{\Gamma} \in \mathbb{R}^{m_{\Gamma}}$ the subvector $\mathbf{v}_{\Gamma}^{i} \in$ $\mathbb{R}^{m_{\Gamma}^{i}}$ whose components correspond to vertices $x_{l} \in \Gamma \cap \partial \Omega_{i}$, i.e., $\mathbf{v}_{\Gamma}^{i}=I_{\Gamma}^{i} \mathbf{v}_{\Gamma}$. 
The optimality conditions (2.6) can now be decomposed into the systems

$$
\begin{gathered}
-\mathbf{M}_{I I}^{i} \frac{\left(\mathbf{p}_{I}^{i}\right)_{k+1}-\left(\mathbf{p}_{I}^{i}\right)_{k}}{\Delta t}+\left(\mathbf{A}_{I I}^{i}\right)^{T}\left(\mathbf{p}_{I}^{i}\right)_{k}+\alpha_{1} \mathbf{M}_{I I}^{i}\left(\mathbf{y}_{I}^{i}\right)_{k} \\
-\mathbf{M}_{I \Gamma}^{i} \frac{\left(\mathbf{p}_{\Gamma}^{i}\right)_{k+1}-\left(\mathbf{p}_{\Gamma}^{i}\right)_{k}}{\Delta t}+\left(\mathbf{A}_{\Gamma I}^{i}\right)^{T}\left(\mathbf{p}_{\Gamma}^{i}\right)_{k}+\alpha_{1} \mathbf{M}_{I \Gamma}^{i}\left(\mathbf{y}_{\Gamma}^{i}\right)_{k}=-\mathbf{c}_{I}^{i}\left(t_{k}\right), \quad k=1, \ldots, K-1 \\
\left(\frac{1}{\Delta t} \mathbf{M}_{I I}^{i}+\left(\mathbf{A}_{I I}^{i}\right)^{T}\right)\left(\mathbf{p}_{I}^{i}\right)_{K}+\left(\alpha_{1}+\frac{\alpha_{2}}{\Delta t}\right) \mathbf{M}_{I I}^{i}\left(\mathbf{y}_{I}^{i}\right)_{K} \\
+\left(\frac{1}{\Delta t} \mathbf{M}_{I \Gamma}^{i}+\left(\mathbf{A}_{\Gamma I}^{i}\right)^{T}\right)\left(\mathbf{p}_{\Gamma}^{i}\right)_{K}+\left(\alpha_{1}+\frac{\alpha_{2}}{\Delta t}\right) \mathbf{M}_{I \Gamma}^{i}\left(\mathbf{y}_{\Gamma}^{i}\right)_{K}=-\mathbf{c}_{I}^{i}\left(t_{K}\right)-\frac{1}{\Delta t} \mathbf{d}_{I}^{i} \\
\alpha_{3} \mathbf{Q}_{I I}\left(\mathbf{u}_{I}^{i}\right)_{k}+\left(\mathbf{B}_{I I}^{i}\right)^{T}\left(\mathbf{p}_{I}^{i}\right)_{k}+\left(\mathbf{B}_{\Gamma I}^{i}\right)^{T}\left(\mathbf{p}_{\Gamma}^{i}\right)_{k}=0, \quad k=1, \ldots, K \\
\mathbf{M}_{I I}^{i} \frac{\left(\mathbf{y}_{I}^{i}\right)_{k}-\left(\mathbf{y}_{I}^{i}\right)_{k-1}}{\Delta t}+\mathbf{A}_{I I}^{i}\left(\mathbf{y}_{I}^{i}\right)_{k}+\mathbf{B}_{I I}^{i}\left(\mathbf{u}_{I}^{i}\right)_{k} \\
+\mathbf{M}_{I \Gamma}^{i} \frac{\left(\mathbf{y}_{\Gamma}^{i}\right)_{k}-\left(\mathbf{y}_{\Gamma}^{i}\right)_{k-1}}{\Delta t}+\mathbf{A}_{I \Gamma}^{i}\left(\mathbf{y}_{\Gamma}^{i}\right)_{k}=\mathbf{f}_{I}^{i}\left(t_{k}\right), \quad k=1, \ldots, K
\end{gathered}
$$

for $i=1, \ldots, s$, where $\left(\mathbf{y}_{I}^{i}\right)_{0}$ is the subvector of the given vector of initial data corresponding to nodes in the interior of $\Omega_{i}$, and into the interface coupling condition

$$
\begin{gathered}
\sum_{i=1}^{s}\left(I_{\Gamma}^{i}\right)^{T}\left(-\mathbf{M}_{\Gamma I}^{i} \frac{\left(\mathbf{p}_{I}^{i}\right)_{k+1}-\left(\mathbf{p}_{I}^{i}\right)_{k}}{\Delta t}+\left(\mathbf{A}_{I \Gamma}^{i}\right)^{T}\left(\mathbf{p}_{I}^{i}\right)_{k}\right. \\
-\mathbf{M}_{\Gamma \Gamma}^{i} \frac{\left(\mathbf{p}_{\Gamma}^{i}\right)_{k+1}-\left(\mathbf{p}_{\Gamma}^{i}\right)_{k}}{\Delta t}+\left(\mathbf{A}_{\Gamma \Gamma}^{i}\right)^{T}\left(\mathbf{p}_{\Gamma}^{i}\right)_{k} \\
\left.+\alpha_{1} \mathbf{M}_{\Gamma I}^{i}\left(\mathbf{y}_{I}^{i}\right)_{k}+\alpha_{1} \mathbf{M}_{\Gamma \Gamma}^{i}\left(\mathbf{y}_{\Gamma}^{i}\right)_{k}\right)=-\mathbf{c}_{\Gamma}\left(t_{k}\right), \quad k=1, \ldots, K-1 \\
\sum_{i=1}^{s}\left(I_{\Gamma}^{i}\right)^{T}\left(\left(\frac{1}{\Delta t} \mathbf{M}_{\Gamma I}^{i}+\left(\mathbf{A}_{I \Gamma}^{i}\right)^{T}\right)\left(\mathbf{p}_{I}^{i}\right)_{K}+\left(\alpha_{1}+\frac{\alpha_{2}}{\Delta t}\right) \mathbf{M}_{\Gamma I}^{i}\left(\mathbf{y}_{I}^{i}\right)_{K}\right. \\
\left.+\left(\frac{1}{\Delta t} \mathbf{M}_{\Gamma \Gamma}^{i}+\left(\mathbf{A}_{\Gamma \Gamma}^{i}\right)^{T}\right)\left(\mathbf{p}_{\Gamma}^{i}\right)_{K}+\left(\alpha_{1}+\frac{\alpha_{2}}{\Delta t}\right) \mathbf{M}_{\Gamma \Gamma}^{i}\left(\mathbf{y}_{\Gamma}^{i}\right)_{K}\right)=-\mathbf{c}_{\Gamma}\left(t_{K}\right)-\frac{1}{\Delta t} \mathbf{d}_{\Gamma} \\
\sum_{i=1}^{s}\left(I_{\Gamma}^{i}\right)^{T}\left(\mathbf{M}_{\Gamma I}^{i} \frac{\left(\mathbf{y}_{I}^{i}\right)_{k}-\left(\mathbf{y}_{I}^{i}\right)_{k-1}}{\Delta t}+\mathbf{A}_{\Gamma I}^{i}\left(\mathbf{y}_{I}^{i}\right)_{k}+\mathbf{B}_{\Gamma I}^{i}\left(\mathbf{u}_{I}^{i}\right)_{k}\right. \\
+\mathbf{M}_{\Gamma \Gamma}^{i} \frac{\left.\left(\mathbf{y}_{\Gamma}^{i}\right)_{k}-\left(\mathbf{y}_{\Gamma}^{i}\right)_{k-1}+\mathbf{A}_{\Gamma \Gamma}^{i}\left(\mathbf{y}_{\Gamma}^{i}\right)_{k}\right)=\mathbf{f}_{\Gamma}\left(t_{k}\right), \quad k=1, \ldots, K}{\Delta t}
\end{gathered}
$$

where $I_{\Gamma}^{i}$ is the matrix defined in (3.1) and where $\left(\mathbf{y}_{\Gamma}^{i}\right)_{0}$ is the subvector of the given vector of initial data corresponding to nodes on $\partial \Omega_{i} \backslash \partial \Omega$.

We now view the solution $\left(\mathbf{y}_{I}^{i}\right)_{k},\left(\mathbf{u}_{I}^{i}\right)_{k},\left(\mathbf{p}_{I}^{i}\right)_{k}, k=1, \ldots, K$, of (3.2) as a function of $\left(\mathbf{y}_{\Gamma}\right)_{1}, \ldots,\left(\mathbf{y}_{\Gamma}\right)_{K}$, $\left(\mathbf{p}_{\Gamma}\right)_{1}, \ldots,\left(\mathbf{p}_{\Gamma}\right)_{K}$. Then (3.3) represents a system of equations in $\left(\mathbf{y}_{\Gamma}\right)_{1}, \ldots,\left(\mathbf{y}_{\Gamma}\right)_{K},\left(\mathbf{p}_{\Gamma}\right)_{1}, \ldots,\left(\mathbf{p}_{\Gamma}\right)_{K}$. Before, we give a precise statement of this system, we give an interpretation of the system (3.2), $i=1, \ldots, s$, as the optimality conditions of an optimal control problem. 
Theorem 3.1 Given $\left(\mathbf{y}_{\Gamma}^{i}\right)_{1}, \ldots,\left(\mathbf{y}_{\Gamma}^{i}\right)_{K},\left(\mathbf{p}_{\Gamma}^{i}\right)_{1}, \ldots,\left(\mathbf{p}_{\Gamma}^{i}\right)_{K}$, the system $(3.2), i=1, \ldots, s$, can be interpreted as the necessary and sufficient optimality conditions for the following discrete subdomain optimal control problems in the unknowns $\left(\mathbf{y}_{I}^{i}\right)_{1}, \ldots,\left(\mathbf{y}_{I}^{i}\right)_{K},\left(\mathbf{u}_{I}^{i}\right)_{1}, \ldots,\left(\mathbf{u}_{I}^{i}\right)_{K}$.

$$
\begin{aligned}
\text { Minimize } \sum_{k=1}^{K} & \frac{\alpha_{1} \Delta t}{2}\left(\mathbf{y}_{I}^{i}\right)_{k}^{T} \mathbf{M}_{I I}^{i}\left(\mathbf{y}_{I}^{i}\right)_{k} \\
& \quad+\Delta t\left(\mathbf{c}_{I}^{i}\left(t_{k}\right)-\mathbf{M}_{I \Gamma}^{i} \frac{\left(\mathbf{p}_{\Gamma}^{i}\right)_{k+1}-\left(\mathbf{p}_{\Gamma}^{i}\right)_{k}}{\Delta t}+\left(\mathbf{A}_{\Gamma I}^{i}\right)^{T}\left(\mathbf{p}_{\Gamma}^{i}\right)_{k}+\alpha_{1} \mathbf{M}_{I \Gamma}^{i}\left(\mathbf{y}_{\Gamma}^{i}\right)_{k}\right)^{T}\left(\mathbf{y}_{I}^{i}\right)_{k} \\
+ & \frac{\alpha_{2}}{2}\left(\mathbf{y}_{I}^{i}\right)_{K}^{T} \mathbf{M}_{I I}^{i}\left(\mathbf{y}_{I}^{i}\right)_{K}+\left(\mathbf{d}_{I}^{i}+\mathbf{M}_{I \Gamma}^{i}\left(\mathbf{p}_{\Gamma}^{i}\right)_{K+1}+\alpha_{2} \mathbf{M}_{I \Gamma}^{i}\left(\mathbf{y}_{\Gamma}^{i}\right)_{K}\right)^{T}\left(\mathbf{y}_{I}^{i}\right)_{K} \\
+ & \sum_{k=1}^{K} \frac{\alpha_{3} \Delta t}{2}\left(\mathbf{u}_{I}^{i}\right)_{k}^{T} \mathbf{Q}_{I I}^{i}\left(\mathbf{u}_{I}^{i}\right)_{k}+\Delta t\left(\mathbf{p}_{\Gamma}^{i}\right)_{k}^{T} \mathbf{B}_{\Gamma I}^{i}\left(\mathbf{u}_{I}^{i}\right)_{k},
\end{aligned}
$$

subject to $\mathbf{M}_{I I}^{i} \frac{\left(\mathbf{y}_{I}^{i}\right)_{k}-\left(\mathbf{y}_{I}^{i}\right)_{k-1}}{\Delta t}+\mathbf{A}_{I I}^{i}\left(\mathbf{y}_{I}^{i}\right)_{k}+\mathbf{B}_{I I}^{i}\left(\mathbf{u}_{I}^{i}\right)_{k}$

$$
=\mathbf{f}_{I}^{i}\left(t_{k}\right)-\mathbf{M}_{I \Gamma}^{i} \frac{\left(\mathbf{y}_{\Gamma}^{i}\right)_{k}-\left(\mathbf{y}_{\Gamma}^{i}\right)_{k-1}}{\Delta t}-\mathbf{A}_{I \Gamma}^{i}\left(\mathbf{y}_{\Gamma}^{i}\right)_{k} \quad k=1, \ldots, K .
$$

Proof: The Lagrangian for (3.4) is given by

$$
\begin{aligned}
& L_{I}^{i}\left(\left(\mathbf{y}_{I}^{i}\right)_{1}, \ldots,\left(\mathbf{y}_{I}^{i}\right)_{K},\left(\mathbf{u}_{I}^{i}\right)_{1}, \ldots,\left(\mathbf{u}_{I}^{i}\right)_{K},\left(\mathbf{p}_{I}^{i}\right)_{1}, \ldots,\left(\mathbf{p}_{I}^{i}\right)_{K}\right) \\
& =\sum_{k=1}^{K} \frac{\alpha_{1} \Delta t}{2}\left(\mathbf{y}_{I}^{i}\right)_{k}^{T} \mathbf{M}_{I I}^{i}\left(\mathbf{y}_{I}^{i}\right)_{k} \\
& \quad+\Delta t\left(\mathbf{c}_{I}^{i}\left(t_{k}\right)-\mathbf{M}_{I \Gamma}^{i} \frac{\left(\mathbf{p}_{\Gamma}^{i}\right)_{k+1}-\left(\mathbf{p}_{\Gamma}^{i}\right)_{k}}{\Delta t}+\left(\mathbf{A}_{\Gamma I}^{i}\right)^{T}\left(\mathbf{p}_{\Gamma}^{i}\right)_{k}+\alpha_{1} \mathbf{M}_{I \Gamma}^{i}\left(\mathbf{y}_{\Gamma}^{i}\right)_{k}\right)^{T}\left(\mathbf{y}_{I}^{i}\right)_{k} \\
& \quad+\frac{\alpha_{2}}{2}\left(\mathbf{y}_{I}^{i}\right)_{K}^{T} \mathbf{M}_{I I}^{i}\left(\mathbf{y}_{I}^{i}\right)_{K}+\left(\mathbf{d}_{I}^{i}+\mathbf{M}_{I \Gamma}^{i}\left(\mathbf{p}_{\Gamma}^{i}\right)_{K+1}+\alpha_{2} \mathbf{M}_{I \Gamma}^{i}\left(\mathbf{y}_{\Gamma}^{i}\right)_{K}\right)^{T}\left(\mathbf{y}_{I}^{i}\right)_{K} \\
& \quad+\sum_{k=1}^{K} \frac{\alpha_{3} \Delta t}{2}\left(\mathbf{u}_{I}^{i}\right)_{k}^{T} \mathbf{Q}_{I I}^{i}\left(\mathbf{u}_{I}^{i}\right)_{k}+\Delta t\left(\mathbf{p}_{\Gamma}^{i}\right)_{k}^{T} \mathbf{B}_{\Gamma I}^{i}\left(\mathbf{u}_{I}^{i}\right)_{k} \\
& \quad+\sum_{k=1}^{K}\left(\mathbf{p}_{I}^{i}\right)_{k}^{T} \Delta t\left(\mathbf{M}_{I I}^{i} \frac{\left(\mathbf{y}_{I}^{i}\right)_{k}-\left(\mathbf{y}_{I}^{i}\right)_{k-1}}{\Delta t}+\mathbf{A}_{I I}^{i}\left(\mathbf{y}_{I}^{i}\right)_{k}+\mathbf{B}_{I I}^{i}\left(\mathbf{u}_{I}^{i}\right)_{k}\right. \\
& \left.\quad-\mathbf{f}_{I}^{i}\left(t_{k}\right)+\mathbf{M}_{I \Gamma}^{i} \frac{\left(\mathbf{y}_{\Gamma}^{i}\right)_{k}-\left(\mathbf{y}_{\Gamma}^{i}\right)_{k-1}}{\Delta t}+\mathbf{A}_{I \Gamma}^{i}\left(\mathbf{y}_{\Gamma}^{i}\right)_{k}\right) .
\end{aligned}
$$

The problem (3.4) is convex, linear-quadratic. The necessary and sufficient optimality conditions for (3.4) are obtained by setting the derivatives of the Lagrangian to zero and are given by (3.2).

As we have stated before, we view the solutions $\left(\mathbf{y}_{I}^{i}\right)_{1}, \ldots,\left(\mathbf{y}_{I}^{i}\right)_{K}, \quad\left(\mathbf{p}_{I}^{i}\right)_{1}, \ldots,\left(\mathbf{p}_{I}^{i}\right)_{K}$, $\left(\mathbf{u}_{I}^{i}\right)_{1}, \ldots,\left(\mathbf{u}_{I}^{i}\right)_{K}$ of $(3.2), i=1, \ldots, s$, as affine linear maps of $\left(\mathbf{y}_{\Gamma}^{i}\right)_{1}, \ldots,\left(\mathbf{y}_{\Gamma}^{i}\right)_{K}$, 
$\left(\mathbf{p}_{\Gamma}^{i}\right)_{1}, \ldots,\left(\mathbf{p}_{\Gamma}^{i}\right)_{K}$, and the view (3.3) as a system of linear equations in $\left(\mathbf{y}_{\Gamma}\right)_{1}, \ldots,\left(\mathbf{y}_{\Gamma}\right)_{K}$, $\left(\mathbf{p}_{\Gamma}\right)_{1}, \ldots,\left(\mathbf{p}_{\Gamma}\right)_{K}$. This leads to the linear map

$$
\mathbf{S}_{i}^{\Delta t}:\left(\mathbb{R}^{m_{\Gamma}^{i}}\right)^{2 K} \rightarrow\left(\mathbb{R}^{m_{\Gamma}^{i}}\right)^{2 K}
$$

defined by

$$
\begin{aligned}
& \mathbf{S}_{i}^{\Delta t}\left(\left(\mathbf{y}_{\Gamma}^{i}\right)_{1}, \ldots,\left(\mathbf{y}_{\Gamma}^{i}\right)_{K},\left(\mathbf{p}_{\Gamma}^{i}\right)_{1}, \ldots,\left(\mathbf{p}_{\Gamma}^{i}\right)_{K}\right) \\
& -\left(\begin{array}{c}
\mathbf{M}_{\Gamma I}^{i} \frac{\left(\mathbf{p}_{I}^{i}\right)_{2}-\left(\mathbf{p}_{I}^{i}\right)_{1}}{\Delta t}+\left(\mathbf{A}_{I \Gamma}^{i}\right)^{T}\left(\mathbf{p}_{I}^{i}\right)_{1} \ldots \\
\ldots-\mathbf{M}_{\Gamma \Gamma}^{i} \frac{\left(\mathbf{p}_{\Gamma}^{i}\right)_{2}-\left(\mathbf{p}_{\Gamma}^{i}\right)_{1}}{\Delta t}+\left(\mathbf{A}_{\Gamma \Gamma}^{i}\right)^{T}\left(\mathbf{p}_{\Gamma}^{i}\right)_{1}+\alpha_{1} \mathbf{M}_{\Gamma I}^{i}\left(\mathbf{y}_{I}^{i}\right)_{1}+\alpha_{1} \mathbf{M}_{\Gamma \Gamma}^{i}\left(\mathbf{y}_{\Gamma}^{i}\right)_{1} \\
\vdots \\
\ldots-\mathbf{M}_{\Gamma \Gamma}^{i} \frac{\left(\mathbf{p}_{\Gamma}^{i}\right)_{K}-\left(\mathbf{p}_{\Gamma}^{i}\right)_{K-1}}{\Delta t}+\left(\mathbf{M}_{\Gamma \Gamma}^{i} \frac{\left(\mathbf{p}_{I}^{i}\right)_{K}-\left(\mathbf{p}_{I}^{i}\right)_{K-1}}{\Delta t}+\left(\mathbf{A}_{\Gamma \Gamma}^{i}\right)^{T}\left(\mathbf{p}_{I}^{i}\right)_{K-1} \ldots\right. \\
\left(\frac{1}{\Delta t} \mathbf{M}_{\Gamma I}^{i}+\left(\mathbf{A}_{I \Gamma}^{i}\right)^{T}\right)\left(\mathbf{p}_{I}^{i}\right)_{K}+\left(\alpha_{1}+\frac{\alpha_{2}}{\Delta t}\right) \mathbf{M}_{\Gamma \Gamma}^{i}\left(\mathbf{y}_{I}^{i}\right)_{K} \ldots \\
\ldots+\left(\frac{1}{\Delta t} \mathbf{M}_{\Gamma \Gamma}^{i}+\left(\mathbf{A}_{\Gamma \Gamma}^{i}\right)^{T}\right)\left(\mathbf{p}_{\Gamma}^{i}\right)_{K}+\left(\alpha_{1}+\frac{\alpha_{2}}{\Delta t} \mathbf{M}_{\Gamma \Gamma}^{i}\left(\mathbf{y}_{\Gamma}^{i}\right)_{K}\right. \\
\mathbf{M}_{\Gamma I}^{i} \frac{\left(\mathbf{y}_{I}^{i}\right)_{1}}{\Delta t}+\mathbf{A}_{\Gamma I}^{i}\left(\mathbf{y}_{I}^{i}\right)_{1}+\mathbf{B}_{\Gamma I}^{i}\left(\mathbf{u}_{I}^{i}\right)_{1} \ldots \\
\ldots+\mathbf{M}_{\Gamma \Gamma}^{i} \frac{\left(\mathbf{y}_{\Gamma}^{i}\right)_{1}}{\Delta t}+\mathbf{A}_{\Gamma \Gamma}^{i}\left(\mathbf{y}_{\Gamma}^{i}\right)_{1} \\
\vdots \\
\mathbf{M}_{\Gamma I}^{i} \frac{\left(\mathbf{y}_{I}^{i}\right)_{K}-\left(\mathbf{y}_{I}^{i}\right)_{K-1}}{\Delta t}+\mathbf{A}_{\Gamma I}^{i}\left(\mathbf{y}_{I}^{i}\right)_{K}+\mathbf{B}_{\Gamma I}^{i}\left(\mathbf{u}_{I}^{i}\right)_{K} \ldots \\
\ldots+\mathbf{M}_{\Gamma \Gamma}^{i} \frac{\left(\mathbf{y}_{\Gamma}^{i}\right)_{K}-\left(\mathbf{y}_{\Gamma}^{i}\right)_{K-1}}{\Delta t}+\mathbf{A}_{\Gamma \Gamma}^{i}\left(\mathbf{y}_{\Gamma}^{i}\right)_{K}
\end{array}\right),
\end{aligned}
$$

where $\left(\mathbf{y}_{I}^{i}\right)_{1}, \ldots,\left(\mathbf{y}_{I}^{i}\right)_{K},\left(\mathbf{p}_{I}^{i}\right)_{1}, \ldots,\left(\mathbf{p}_{I}^{i}\right)_{K},\left(\mathbf{u}_{I}^{i}\right)_{1}, \ldots,\left(\mathbf{u}_{I}^{i}\right)_{K}$ is the solution of (3.2) (or, equivalently, of (3.4)) with $\left(\mathbf{y}_{0}\right)_{I}^{i}=\mathbf{0}, \mathbf{f}_{I}^{i}=\mathbf{0}, \mathbf{c}_{I}^{i}=\mathbf{0}, \mathbf{d}_{I}^{i}=\mathbf{0}$. Furthermore, we define

$$
\mathbf{r}_{i}^{\Delta t} \in\left(\mathbb{R}_{\Gamma}^{m_{\Gamma}^{i}}\right)^{2 K}
$$


by

$$
\mathbf{r}_{i}^{\Delta t}=\left(\begin{array}{c}
-\mathbf{c}_{\Gamma}^{i}\left(t_{1}\right)+\mathbf{M}_{\Gamma I}^{i} \frac{\left(\mathbf{p}_{I}^{i}\right)_{2}-\left(\mathbf{p}_{I}^{i}\right)_{1}}{\Delta t}-\left(\mathbf{A}_{I \Gamma}^{i}\right)^{T}\left(\mathbf{p}_{I}^{i}\right)_{1}-\alpha_{1} \mathbf{M}_{\Gamma I}^{i}\left(\mathbf{y}_{I}^{i}\right)_{1} \\
\vdots \\
-\mathbf{c}_{\Gamma}^{i}\left(t_{K-1}\right)+\mathbf{M}_{\Gamma I}^{i} \frac{\left(\mathbf{p}_{I}^{i}\right)_{K}-\left(\mathbf{p}_{I}^{i}\right)_{K-1}}{\Delta t}-\left(\mathbf{A}_{I \Gamma}^{i}\right)^{T}\left(\mathbf{p}_{I}^{i}\right)_{K-1}-\alpha_{1} \mathbf{M}_{\Gamma I}^{i}\left(\mathbf{y}_{I}^{i}\right)_{K-1} \\
-\mathbf{c}_{\Gamma}^{i}\left(t_{K}\right)-\frac{1}{\Delta t} \mathbf{d}_{\Gamma}^{i}-\left(\frac{1}{\Delta t} \mathbf{M}_{\Gamma I}^{i}+\left(\mathbf{A}_{I \Gamma}^{i}\right)^{T}\right)\left(\mathbf{p}_{I}^{i}\right)_{K}-\left(\alpha_{1}+\frac{\alpha_{2}}{\Delta t}\right) \mathbf{M}_{\Gamma I}^{i}\left(\mathbf{y}_{I}^{i}\right)_{K} \\
\mathbf{f}_{\Gamma}^{i}\left(t_{1}\right)-\mathbf{M}_{\Gamma I}^{i} \frac{\left(\mathbf{y}_{I}^{i}\right)_{1}-\left(\mathbf{y}_{I}^{i}\right)_{0}}{\Delta t}-\mathbf{A}_{\Gamma I}^{i}\left(\mathbf{y}_{I}^{i}\right)_{1}+\mathbf{B}_{\Gamma I}^{i}\left(\mathbf{u}_{I}^{i}\right)_{1}+\mathbf{M}_{\Gamma \Gamma}^{i} \frac{\left(\mathbf{y}_{\Gamma}^{i}\right)_{0}}{\Delta t} \\
\vdots \\
\mathbf{f}_{\Gamma}^{i}\left(t_{K}\right)-\mathbf{M}_{\Gamma I}^{i} \frac{\left(\mathbf{y}_{I}^{i}\right)_{K}-\left(\mathbf{y}_{I}^{i}\right)_{K-1}}{\Delta t}-\mathbf{A}_{\Gamma I}^{i}\left(\mathbf{y}_{I}^{i}\right)_{K}+\mathbf{B}_{\Gamma I}^{i}\left(\mathbf{u}_{I}^{i}\right)_{K}
\end{array}\right),
$$

where $\left(\mathbf{y}_{I}^{i}\right)_{1}, \ldots,\left(\mathbf{y}_{I}^{i}\right)_{K},\left(\mathbf{p}_{I}^{i}\right)_{1}, \ldots,\left(\mathbf{p}_{I}^{i}\right)_{K},\left(\mathbf{u}_{I}^{i}\right)_{1}, \ldots,\left(\mathbf{u}_{I}^{i}\right)_{K}$ is the solution of (3.2) (or, equivalently, of (3.4)) with $\left(\mathbf{y}_{\Gamma}^{i}\right)_{1}=\ldots=\left(\mathbf{y}_{\Gamma}^{i}\right)_{K}=\left(\mathbf{p}_{\Gamma}^{i}\right)_{1}=\ldots=\left(\mathbf{p}_{\Gamma}^{i}\right)_{K}=\mathbf{0}$.

Let $\mathbf{I}_{\Gamma}^{i, \Delta t}=\operatorname{diag}\left(I_{\Gamma}^{i}, \ldots, I_{\Gamma}^{i}\right) \in \mathbb{R}^{2 K m_{\Gamma}^{i} \times 2 K m_{\Gamma}^{i}}$, where $I_{\Gamma}^{i}$ is defined in (3.1). The system (3.2), (3.3a) can now be written as an operator equation

$$
\sum_{i=1}^{s}\left(\mathbf{I}_{\Gamma}^{i, \Delta t}\right)^{T} \mathbf{S}_{i}^{\Delta t} \mathbf{I}_{\Gamma}^{i, \Delta t}\left(\left(\mathbf{y}_{\Gamma}\right)_{1}, \ldots,\left(\mathbf{y}_{\Gamma}\right)_{K},\left(\mathbf{p}_{\Gamma}\right)_{1}, \ldots,\left(\mathbf{p}_{\Gamma}\right)_{K}\right)=\sum_{i=1}^{s}\left(\mathbf{I}_{\Gamma}^{i, \Delta t}\right)^{T} \mathbf{r}_{i}
$$

in the unknowns $\left(\mathbf{y}_{\Gamma}\right)_{1}, \ldots,\left(\mathbf{y}_{\Gamma}\right)_{K},\left(\mathbf{p}_{\Gamma}\right)_{1}, \ldots,\left(\mathbf{p}_{\Gamma}\right)_{K} \in \mathbb{R}^{m_{\Gamma}}$. If the solution $\left(\mathbf{y}_{\Gamma}\right)_{1}, \ldots,\left(\mathbf{y}_{\Gamma}\right)_{K},\left(\mathbf{p}_{\Gamma}\right)_{1}, \ldots,\left(\mathbf{p}_{\Gamma}\right)_{K}$ of (3.7) is computed, then the remaining components $\left(\mathbf{y}_{I}^{i}\right)_{1}, \ldots,\left(\mathbf{y}_{I}^{i}\right)_{K},\left(\mathbf{p}_{I}^{i}\right)_{1}, \ldots,\left(\mathbf{p}_{I}^{i}\right)_{K},\left(\mathbf{u}_{I}^{i}\right)_{1}, \ldots,\left(\mathbf{u}_{I}^{i}\right)_{K}, i=1, \ldots, s$, can be computed by solving (3.2) (or, equivalently, (3.4)).

In the next theorem, we will show how to apply the inverse of the subdomain operator $\mathbf{S}_{i}$, $i=1, \ldots, s$. For this result it is useful to introduce the notation

$$
\mathbf{A}^{i}=\left(\begin{array}{cc}
\mathbf{A}_{I I}^{i} & \mathbf{A}_{I \Gamma}^{i} \\
\mathbf{A}_{\Gamma I}^{i} & \mathbf{A}_{\Gamma \Gamma}^{i}
\end{array}\right), \quad \mathbf{M}^{i}=\left(\begin{array}{cc}
\mathbf{M}_{I I}^{i} & \mathbf{M}_{I \Gamma}^{i} \\
\mathbf{M}_{\Gamma I}^{i} & \mathbf{M}_{\Gamma \Gamma}^{i}
\end{array}\right), \quad \mathbf{B}^{i}=\left(\begin{array}{c}
\mathbf{B}_{I I}^{i} \\
\mathbf{B}_{\Gamma I}^{i}
\end{array}\right)
$$

and

$$
\mathbf{y}_{k}^{i}=\left(\begin{array}{c}
\left(\mathbf{y}_{I}^{i}\right)_{k} \\
\left(\mathbf{y}_{\Gamma}^{i}\right)_{k}
\end{array}\right), \quad \mathbf{p}_{k}^{i}=\left(\begin{array}{c}
\left(\mathbf{p}_{I}^{i}\right)_{k} \\
\left(\mathbf{p}_{\Gamma}^{i}\right)_{k}
\end{array}\right)
$$

Furthermore, let

$$
I^{i} \in \mathbb{R}^{m_{\Gamma}^{i} \times m^{i}}
$$

be the matrix with zero or one entries that extracts out of a vector $\mathbf{v}^{i} \in \mathbb{R}^{m^{i}}$ the subvector $\mathbf{v}_{\Gamma}^{i} \in \mathbb{R}_{\Gamma}^{m_{\Gamma}^{i}}$ whose components correspond to vertices $x_{l} \in \Gamma \cap \partial \Omega_{i}$. 
Theorem 3.2 Let $\mathbf{r}^{i}=\left(\mathbf{r}_{1}^{i}, \ldots, \mathbf{r}_{2 K}^{i}\right) \in\left(\mathbb{R}_{\Gamma}^{m_{\Gamma}^{i}}\right)^{2 K}$ be given. The solution $\left(\mathbf{y}_{\Gamma}^{i}\right)_{1}, \ldots,\left(\mathbf{y}_{\Gamma}^{i}\right)_{K},\left(\mathbf{p}_{\Gamma}^{i}\right)_{1}, \ldots,\left(\mathbf{p}_{\Gamma}^{i}\right)_{K} \in \mathbb{R}^{m_{\Gamma}}$ of

$$
\mathbf{S}_{i}^{\Delta t}\left(\left(\mathbf{y}_{\Gamma}^{i}\right)_{1}, \ldots,\left(\mathbf{y}_{\Gamma}^{i}\right)_{K},\left(\mathbf{p}_{\Gamma}^{i}\right)_{1}, \ldots,\left(\mathbf{p}_{\Gamma}^{i}\right)_{K}\right)=\mathbf{r}_{i}
$$

is given by

$$
\left(\mathbf{y}_{\Gamma}^{i}\right)_{k}=I^{i} \mathbf{y}_{k}^{i}, \quad k=1, \ldots, K, \quad\left(\mathbf{p}_{\Gamma}^{i}\right)_{k}=I^{i} \mathbf{p}_{k}^{i}, \quad k=1, \ldots, K,
$$

where $I^{i}$ is the matrix defined in (3.9) and where $\mathbf{y}_{k}^{i}, \mathbf{u}_{k}^{i}, \mathbf{p}_{k}^{i}, k=1, \ldots, K$, solve

$$
\begin{array}{rlrl}
-\mathbf{M}^{i} \frac{\mathbf{p}_{k+1}^{i}-\mathbf{p}_{k}^{i}}{\Delta t}+\left(\mathbf{A}^{i}\right)^{T} \mathbf{p}_{k}^{i}+\alpha_{1} \mathbf{M}^{i} \mathbf{y}_{k}^{i} & =\left(\begin{array}{c}
\mathbf{0} \\
\mathbf{r}_{k}^{i}
\end{array}\right), & k=1, \ldots, K-1, \\
\left(\frac{1}{\Delta t} \mathbf{M}^{i}+\left(\mathbf{A}^{i}\right)^{T}\right) \mathbf{p}_{K}^{i}+\left(\alpha_{1}+\frac{\alpha_{2}}{\Delta t}\right) \mathbf{M}^{i} \mathbf{y}_{K}^{i} & =\left(\begin{array}{c}
\mathbf{0} \\
\mathbf{r}_{K}^{i}
\end{array}\right), & & \\
\alpha_{3} \mathbf{Q}_{I I}\left(\mathbf{u}_{I}^{i}\right)_{k}+\left(\mathbf{B}^{i}\right)^{T} \mathbf{p}_{k}^{i} & =\mathbf{0}, & k=1, \ldots, K \\
\mathbf{M}^{i} \frac{\mathbf{y}_{k}^{i}-\mathbf{y}_{k-1}^{i}}{\Delta t}+\mathbf{A}^{i} \mathbf{y}_{k}^{i}+\mathbf{B}^{i} \mathbf{u}_{k}^{i} & =\left(\begin{array}{c}
\mathbf{0} \\
\mathbf{r}_{K+k}^{i}
\end{array}\right), & k=1, \ldots, K \\
\mathbf{y}_{0}^{i} & =\mathbf{0} .
\end{array}
$$

The equations (3.10) are the system of necessary and sufficient optimality conditions for the optimal control problem

$$
\begin{aligned}
& \text { minimize } \sum_{k=1}^{K} \frac{\alpha_{1} \Delta t}{2}\left(\mathbf{y}_{k}^{i}\right)^{T} \mathbf{M}^{i} \mathbf{y}_{k}^{i}-\Delta t\left(\mathbf{0}^{T},\left(\mathbf{r}_{k}^{i}\right)^{T}\right) \mathbf{y}_{k}^{i} \\
&+ \frac{\alpha_{2}}{2}\left(\mathbf{y}_{K}^{i}\right)^{T} \mathbf{M}^{i} \mathbf{y}_{K}^{i}+\sum_{k=1}^{K} \frac{\alpha_{3} \Delta t}{2}\left(\mathbf{u}_{I}^{i}\right)_{k}^{T} \mathbf{Q}_{I I}^{i}\left(\mathbf{u}_{I}^{i}\right)_{k}, \\
& \text { subject to } \mathbf{M}^{i} \frac{\mathbf{y}_{k}^{i}-\mathbf{y}_{k-1}^{i}}{\Delta t}+\mathbf{A}^{i} \mathbf{y}_{k}^{i}+\mathbf{B}^{i} \mathbf{u}_{k}^{i}=\left(\begin{array}{c}
\mathbf{0} \\
\mathbf{r}_{K+k}^{i}
\end{array}\right), \quad k=1, \ldots, K, \\
& \mathbf{y}_{0}^{i}=\mathbf{0}
\end{aligned}
$$

Proof: By definition (3.5) of $\mathbf{S}_{i}$ the equation

$$
\mathbf{S}_{i}^{\Delta t}\left(\left(\mathbf{y}_{\Gamma}^{i}\right)_{1}, \ldots,\left(\mathbf{y}_{\Gamma}^{i}\right)_{K},\left(\mathbf{p}_{\Gamma}^{i}\right)_{1}, \ldots,\left(\mathbf{p}_{\Gamma}^{i}\right)_{K}\right)=\mathbf{r}^{i}
$$


is equivalent to

$$
\begin{aligned}
& -\mathbf{M}_{I I}^{i} \frac{\left(\mathbf{p}_{I}^{i}\right)_{k+1}-\left(\mathbf{p}_{I}^{i}\right)_{k}}{\Delta t}+\left(\mathbf{A}_{I I}^{i}\right)^{T}\left(\mathbf{p}_{I}^{i}\right)_{k}+\alpha_{1} \mathbf{M}_{I I}^{i}\left(\mathbf{y}_{I}^{i}\right)_{k} \\
& -\mathbf{M}_{I \Gamma}^{i} \frac{\left(\mathbf{p}_{\Gamma}^{i}\right)_{k+1}-\left(\mathbf{p}_{\Gamma}^{i}\right)_{k}}{\Delta t}+\left(\mathbf{A}_{\Gamma I}^{i}\right)^{T}\left(\mathbf{p}_{\Gamma}^{i}\right)_{k}+\alpha_{1} \mathbf{M}_{I \Gamma}^{i}\left(\mathbf{y}_{\Gamma}^{i}\right)_{k}=\mathbf{0}, \quad k=1, \ldots, K-1 \\
& \left(\frac{1}{\Delta t} \mathbf{M}_{I I}^{i}+\left(\mathbf{A}_{I I}^{i}\right)^{T}\right)\left(\mathbf{p}_{I}^{i}\right)_{K}+\left(\alpha_{1}+\frac{\alpha_{2}}{\Delta t}\right) \mathbf{M}_{I I}^{i}\left(\mathbf{y}_{I}^{i}\right)_{K} \\
& +\left(\frac{1}{\Delta t} \mathbf{M}_{I \Gamma}^{i}+\left(\mathbf{A}_{\Gamma I}^{i}\right)^{T}\right)\left(\mathbf{p}_{\Gamma}^{i}\right)_{K}+\left(\alpha_{1}+\frac{\alpha_{2}}{\Delta t}\right) \mathbf{M}_{I \Gamma}^{i}\left(\mathbf{y}_{\Gamma}^{i}\right)_{K}=\mathbf{0} \\
& \alpha_{3} \mathbf{Q}_{I I}\left(\mathbf{u}_{I}^{i}\right)_{k}+\left(\mathbf{B}_{I I}^{i}\right)^{T}\left(\mathbf{p}_{I}^{i}\right)_{k}+\left(\mathbf{B}_{\Gamma I}^{i}\right)^{T}\left(\mathbf{p}_{\Gamma}^{i}\right)_{k}=\mathbf{0}, \quad k=1, \ldots, K, \\
& \mathbf{M}_{I I}^{i} \frac{\left(\mathbf{y}_{I}^{i}\right)_{k}-\left(\mathbf{y}_{I}^{i}\right)_{k-1}}{\Delta t}+\mathbf{A}_{I I}^{i}\left(\mathbf{y}_{I}^{i}\right)_{k}+\mathbf{B}_{I I}^{i}\left(\mathbf{u}_{I}^{i}\right)_{k} \\
& +\mathbf{M}_{I \Gamma}^{i} \frac{\left(\mathbf{y}_{\Gamma}^{i}\right)_{k}-\left(\mathbf{y}_{\Gamma}^{i}\right)_{k-1}}{\Delta t}+\mathbf{A}_{I \Gamma}^{i}\left(\mathbf{y}_{\Gamma}^{i}\right)_{k}=\mathbf{0}, \quad k=1, \ldots, K, \\
& \left(\mathbf{y}_{I}^{i}\right)_{0}=\mathbf{0},
\end{aligned}
$$

and

$$
\begin{gathered}
-\mathbf{M}_{\Gamma I}^{i} \frac{\left(\mathbf{p}_{I}^{i}\right)_{k+1}-\left(\mathbf{p}_{I}^{i}\right)_{k}}{\Delta t}+\left(\mathbf{A}_{I \Gamma}^{i}\right)^{T}\left(\mathbf{p}_{I}^{i}\right)_{k} \\
-\mathbf{M}_{\Gamma \Gamma}^{i} \frac{\left(\mathbf{p}_{\Gamma}^{i}\right)_{k+1}-\left(\mathbf{p}_{\Gamma}^{i}\right)_{k}}{\Delta t}+\left(\mathbf{A}_{\Gamma \Gamma}^{i}\right)^{T}\left(\mathbf{p}_{\Gamma}^{i}\right)_{k} \\
+\alpha_{1} \mathbf{M}_{\Gamma I}^{i}\left(\mathbf{y}_{I}^{i}\right)_{k}+\alpha_{1} \mathbf{M}_{\Gamma \Gamma}^{i}\left(\mathbf{y}_{\Gamma}^{i}\right)_{k}=\mathbf{r}_{k}^{i}, \quad k=1, \ldots, K-1, \\
\left(\frac{1}{\Delta t} \mathbf{M}_{\Gamma I}^{i}+\left(\mathbf{A}_{I \Gamma}^{i}\right)^{T}\right)\left(\mathbf{p}_{I}^{i}\right)_{K}+\left(\alpha_{1}+\frac{\alpha_{2}}{\Delta t}\right) \mathbf{M}_{\Gamma I}^{i}\left(\mathbf{y}_{I}^{i}\right)_{K} \\
+\left(\frac{1}{\Delta t} \mathbf{M}_{\Gamma \Gamma}^{i}+\left(\mathbf{A}_{\Gamma \Gamma}^{i}\right)^{T}\right)\left(\mathbf{p}_{\Gamma}^{i}\right)_{K}+\left(\alpha_{1}+\frac{\alpha_{2}}{\Delta t}\right) \mathbf{M}_{\Gamma \Gamma}^{i}\left(\mathbf{y}_{\Gamma}^{i}\right)_{K}=\mathbf{r}_{K}^{i}, \\
\mathbf{M}_{\Gamma I}^{i} \frac{\left(\mathbf{y}_{I}^{i}\right)_{k}-\left(\mathbf{y}_{I}^{i}\right)_{k-1}}{\Delta t}+\mathbf{A}_{\Gamma I}^{i}\left(\mathbf{y}_{I}^{i}\right)_{k}+\mathbf{B}_{\Gamma I}^{i}\left(\mathbf{u}_{I}^{i}\right)_{k} \\
+\mathbf{M}_{\Gamma \Gamma}^{i} \frac{\left(\mathbf{y}_{\Gamma}^{i}\right)_{k}-\left(\mathbf{y}_{\Gamma}^{i}\right)_{k-1}}{\Delta t}+\mathbf{A}_{\Gamma \Gamma}^{i}\left(\mathbf{y}_{\Gamma}^{i}\right)_{k}=\mathbf{r}_{K+k}^{i}, \quad k=1, \ldots, K, \\
\left(\mathbf{y}_{\Gamma}^{i}\right)_{0}=\mathbf{0},
\end{gathered}
$$

Using the notation (3.8), the system (3.12) can be written in the compact form (3.10).

The proof that (3.10) are the necessary and sufficient optimality conditions for (3.11) can be carried out analogously the proofs of Theorem 2.2 or Theorem 3.1. We omit the details.

Theorem 3.3 The matrices $\mathbf{S}_{i}^{\Delta t}, i=1, \ldots, s$, defined in (3.5) are symmetric. 
Proof: We define

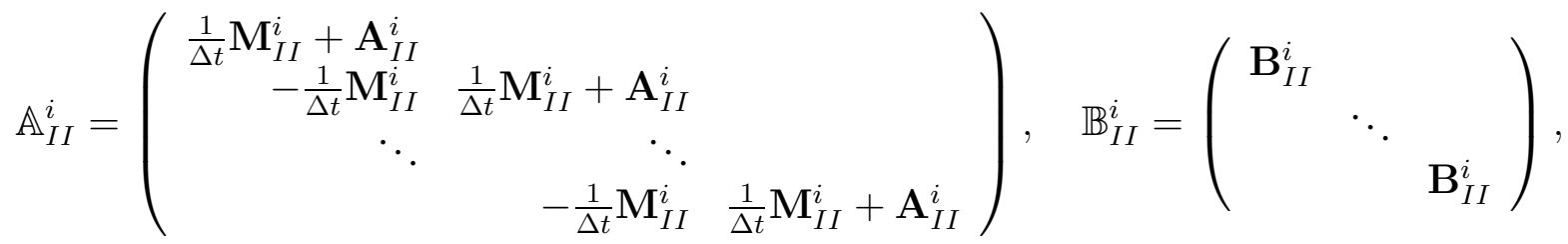

$$
\begin{aligned}
& \mathbb{M}_{I I}^{i}=\left(\begin{array}{cccc}
\alpha_{1} \mathbf{M}_{I I}^{i} & & & \\
& \ddots & & \\
& & \alpha_{1} \mathbf{M}_{I I}^{i} & \\
& & & \left(\alpha_{1}+\frac{\alpha_{2}}{\Delta t}\right) \mathbf{M}_{I I}^{i}
\end{array}\right), \quad \mathbb{Q}_{I I}^{i}=\left(\begin{array}{ccc}
\mathbf{Q}_{I I}^{i} & & \\
& \ddots & \\
& & \mathbf{Q}_{I I}^{i}
\end{array}\right) .
\end{aligned}
$$

The matrices $\mathbb{A}_{\Gamma I}^{i}, \mathbb{B}_{\Gamma I}^{i}$, etc., are defined analogously. We set

$$
\mathbf{x}_{I}^{i}=\left(\left(\mathbf{y}_{I}^{i}\right)_{1}^{T}, \ldots,\left(\mathbf{y}_{I}^{i}\right)_{K}^{T},\left(\mathbf{u}_{I}^{i}\right)_{1}^{T}, \ldots,\left(\mathbf{u}_{I}^{i}\right)_{K}^{T},\left(\mathbf{p}_{I}^{i}\right)_{1}^{T}, \ldots,\left(\mathbf{p}_{I}^{i}\right)_{K}^{T}\right)^{T}
$$

and

$$
\mathbf{x}_{\Gamma}^{i}=\left(\left(\mathbf{y}_{\Gamma}^{i}\right)_{1}^{T}, \ldots,\left(\mathbf{y}_{\Gamma}^{i}\right)_{K}^{T},\left(\mathbf{p}_{\Gamma}^{i}\right)_{1}^{T}, \ldots,\left(\mathbf{p}_{\Gamma}^{i}\right)_{K}^{T}\right)^{T} .
$$

With this notation, the solution $\left(\mathbf{y}_{I}^{i}\right)_{1}, \ldots,\left(\mathbf{y}_{I}^{i}\right)_{K},\left(\mathbf{p}_{I}^{i}\right)_{1}, \ldots,\left(\mathbf{p}_{I}^{i}\right)_{K},\left(\mathbf{u}_{I}^{i}\right)_{1}, \ldots,\left(\mathbf{u}_{I}^{i}\right)_{K}$ of (3.2) with $\left(\mathbf{y}_{0}\right)_{I}^{i}=\mathbf{0}, \mathbf{f}_{I}^{i}=\mathbf{0}, \mathbf{c}_{I}^{i}=\mathbf{0}, \mathbf{d}_{I}^{i}=\mathbf{0}$ is given by

$$
\mathbf{x}_{I}^{i}=-\left(\begin{array}{ccc}
\mathbb{M}_{I I}^{i} & & \left(\mathbb{A}_{I I}^{i}\right)^{T} \\
& \mathbb{Q}_{I I}^{i} & \left(\mathbb{B}_{I I}^{i}\right)^{T} \\
\mathbb{A}_{I I}^{i} & \mathbb{B}_{I I}^{i} &
\end{array}\right)^{-1}\left(\begin{array}{cc}
\mathbb{M}_{I \Gamma}^{i} & \left(\mathbb{A}_{\Gamma I}^{i}\right)^{T} \\
& \left(\mathbb{B}_{\Gamma I}^{i}\right)^{T} \\
\mathbb{A}_{I \Gamma}^{i} &
\end{array}\right) \mathbf{x}_{\Gamma}^{i}
$$

Furthermore,

$$
\begin{aligned}
& \mathbf{S}_{i}^{\Delta t}\left(\mathbf{x}_{\Gamma}^{i}\right)=\left(\begin{array}{cc}
\mathbb{M}_{\Gamma \Gamma}^{i} & \left(\mathbb{A}_{\Gamma \Gamma}^{i}\right)^{T} \\
\mathbb{A}_{\Gamma \Gamma}^{i} &
\end{array}\right) \mathbf{x}_{\Gamma}^{i}+\left(\begin{array}{ccc}
\mathbb{M}_{\Gamma I}^{i} & & \left(\mathbb{A}_{I \Gamma}^{i}\right)^{T} \\
\mathbb{A}_{\Gamma I}^{i} & \mathbb{B}_{\Gamma I}^{i} &
\end{array}\right) \mathbf{x}_{I}^{i}, \\
& =\left[\left(\begin{array}{cc}
\mathbb{M}_{\Gamma \Gamma}^{i} & \left(\mathbb{A}_{\Gamma \Gamma}^{i}\right)^{T} \\
\mathbb{A}_{\Gamma \Gamma}^{i} &
\end{array}\right)\right. \\
& \left.-\left(\begin{array}{ccc}
\mathbb{M}_{\Gamma I}^{i} & & \left(\mathbb{A}_{I \Gamma}^{i}\right)^{T} \\
\mathbb{A}_{\Gamma I}^{i} & \mathbb{B}_{\Gamma I}^{i} &
\end{array}\right)\left(\begin{array}{lll}
\mathbb{M}_{I I}^{i} & & \left(\mathbb{A}_{I I}^{i}\right)^{T} \\
& \mathbb{Q}_{I I}^{i} & \left(\mathbb{B}_{I I}^{i}\right)^{T} \\
\mathbb{A}_{I I}^{i} & \mathbb{B}_{I I}^{i} &
\end{array}\right)^{-1}\left(\begin{array}{cc}
\mathbb{M}_{I \Gamma}^{i} & \left(\mathbb{A}_{\Gamma I}^{i}\right)^{T} \\
& \left(\mathbb{B}_{\Gamma I}^{i}\right)^{T} \\
\mathbb{A}_{I \Gamma}^{i} &
\end{array}\right)\right] \mathbf{x}_{\Gamma}^{i} .
\end{aligned}
$$

The last identity reveals the symmetry of $\mathbf{S}_{i}^{\Delta t}$.

\subsection{Solution Algorithm}

In the previous section we have shown that the discrete optimal control problem (2.5) is equivalent to the linear operator equation (3.7). We solve (3.7) using preconditioned GMRES [9, 19, 22] 
or the symmetric QMR (sQMR) [7, 8]. The inverse of the system operator $\sum_{i=1}^{s}\left(\mathbf{I}_{\Gamma}^{i, \Delta t}\right)^{T} \mathbf{S}_{i}^{\Delta t} \mathbf{I}_{\Gamma}^{i, \Delta t}$ is approximated by a weighted sum of inverses of the subdomain operators $\mathbf{S}_{i}^{\Delta t}$. This choice is motivated by Neumann-Neumann domain decomposition preconditioners that have been used sucessfully for the solution of elliptic PDEs (see $[18,20,21]$ and the references given therein) as well as of elliptic linear-quadratic optimal control problems $[11,12]$. We let $D_{\Gamma}^{i} \in \mathbb{R}^{m_{\Gamma}^{i} \times m_{\Gamma}^{i}}$ be positive definite diagonal matrices such that

$$
\sum_{i=1}^{s}\left(\mathbf{I}_{\Gamma}^{i}\right)^{T} D_{\Gamma}^{i}=\mathbf{I}
$$

In our case the entry $\left(D_{\Gamma}^{i}\right)_{l l}$ is equal to one over the number of subdomains containing the interface node $x_{l}$. We set

$$
\mathbf{D}_{\Gamma}^{i, \Delta t}=\operatorname{diag}\left(D_{\Gamma}^{i}, \ldots, D_{\Gamma}^{i}\right) \in \mathbb{R}^{2 K m_{\Gamma}^{i} \times 2 K m_{\Gamma}^{i}} .
$$

The preconditioner for $\sum_{i=1}^{s}\left(\mathbf{I}_{\Gamma}^{i, \Delta t}\right)^{T} \mathbf{S}_{i}^{\Delta t} \mathbf{I}_{\Gamma}^{i, \Delta t}$ is now given by

$$
\mathbf{P}^{\Delta t}=\sum_{i=1}^{s}\left(\mathbf{I}_{\Gamma}^{i, \Delta t}\right)^{T} \mathbf{D}_{\Gamma}^{i, \Delta t}\left(\mathbf{S}_{i}^{\Delta t}\right)^{-1} \mathbf{D}_{\Gamma}^{i, \Delta t} \mathbf{I}_{\Gamma}^{i, \Delta t}
$$

The unknowns $\left(\mathbf{y}_{\Gamma}\right)_{1}, \ldots,\left(\mathbf{y}_{\Gamma}\right)_{K},\left(\mathbf{p}_{\Gamma}\right)_{1}, \ldots,\left(\mathbf{p}_{\Gamma}\right)_{K} \in \mathbb{R}^{m_{\Gamma}}$ to be determined via GMRES are the discretizations of the states and the adjoints on the subdomain interfaces $\Gamma \times(0, T)$. The evaluation of a matrix vector product

$$
\sum_{i=1}^{s}\left(\mathbf{I}_{\Gamma}^{i, \Delta t}\right)^{T} \mathbf{S}_{i}^{\Delta t} \mathbf{I}_{\Gamma}^{i, \Delta t}\left(\left(\mathbf{y}_{\Gamma}\right)_{1}, \ldots,\left(\mathbf{y}_{\Gamma}\right)_{K},\left(\mathbf{p}_{\Gamma}\right)_{1}, \ldots,\left(\mathbf{p}_{\Gamma}\right)_{K}\right)
$$

can be done in parallel on $s$ processors. Each processor has to evaluate

$$
\mathbf{S}_{i}^{\Delta t} \mathbf{I}_{\Gamma}^{i, \Delta t}\left(\left(\mathbf{y}_{\Gamma}\right)_{1}, \ldots,\left(\mathbf{y}_{\Gamma}\right)_{K},\left(\mathbf{p}_{\Gamma}\right)_{1}, \ldots,\left(\mathbf{p}_{\Gamma}\right)_{K}\right)
$$

which corresponds to the solution of a subdomain optimal control problem (3.4) (cf. Theorems 3.1). These subdomain optimal control problems can be solved using standard techniques and their solution only involves discretizations of states, controls and adjoints on the smaller domains $\Omega_{i} \times(0, T)$. Similarly, the application of the preconditioner (3.14) to a vector $\left(\mathbf{v}_{\Gamma}\right)_{1}, \ldots,\left(\mathbf{v}_{\Gamma}\right)_{K},\left(\mathbf{q}_{\Gamma}\right)_{1}, \ldots,\left(\mathbf{q}_{\Gamma}\right)_{K+1} \in \mathbb{R}^{m_{\Gamma}}$ can be done in parallel on $s$ processors. Each processor has to evaluate

$$
\left(\mathbf{S}_{i}^{\Delta t}\right)^{-1} \mathbf{D}_{\Gamma}^{i, \Delta t} \mathbf{I}_{\Gamma}^{i, \Delta t}\left(\left(\mathbf{v}_{\Gamma}\right)_{1}, \ldots,\left(\mathbf{v}_{\Gamma}\right)_{K},\left(\mathbf{q}_{\Gamma}\right)_{1}, \ldots,\left(\mathbf{q}_{\Gamma}\right)_{K}\right),
$$

which corresponds to the solution of a subdomain optimal control problem (3.11) (cf. Theorem 3.2). Again, these subdomain optimal control problems can be solved using standard techniques and their solution only involves discretizations of states, controls and adjoints on the smaller domains $\Omega_{i} \times(0, T)$. 


\section{Numerical Results}

We consider (1.1) with $\Omega=(0,1), f=0$ and $y_{0}(x)=\sin (2 \pi x)$. The desired states $\hat{y}$ and $\hat{y}_{T}$ are given by the hat functions $\hat{y}(x, t)=\min \{2 x, 2(1-x)\}$ and $\hat{y}_{T}(x)=\min \{2 x, 2(1-x)\}$, respectively. For the spatial discretization of the problem we use piecewise linear finite elements on an equidistant grid with mesh size $\Delta x=1 / K$ and for the time discretization, we use the backward Euler method with step size $\Delta t=1 / K$. The domain $\Omega=(0,1)$ is subdivided into equidistant subdomains $\Omega_{i}=((i-1) H, i H), i=1, \ldots, s, H=1 / s$. For $\alpha_{1}=\alpha_{2}=10^{3}, \alpha_{3}=1$, and $s=4$, the computed optimal control and corresponding state are shown in Figure 4.1.
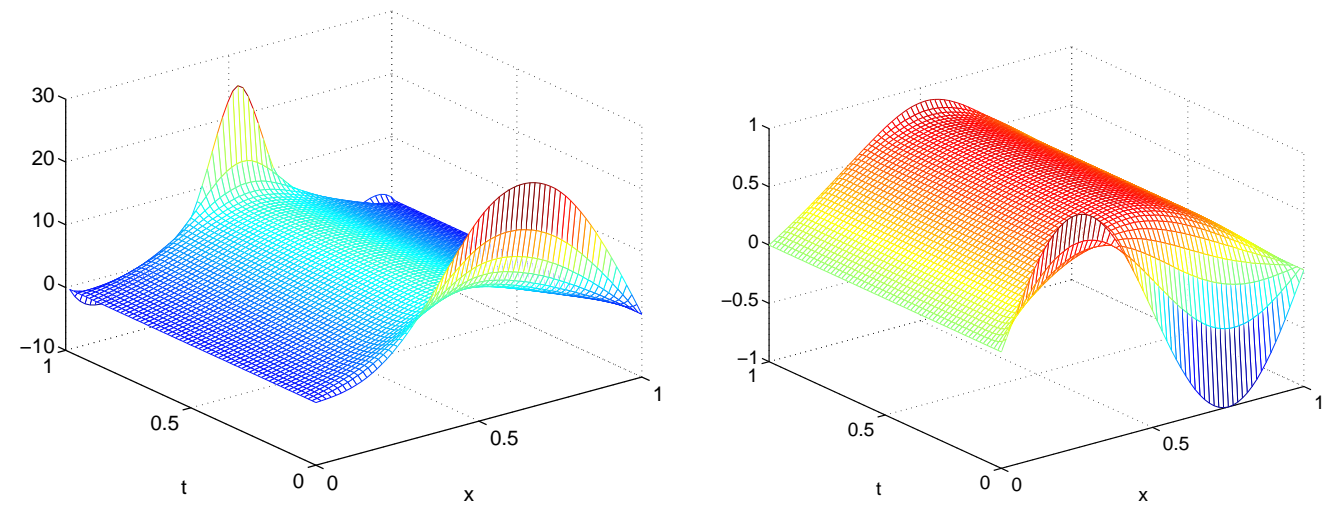

Figure 4.1: Computed optimal control (left) and corresponding state (right) for the example problem with $\alpha_{1}=\alpha_{2}=10^{3}, \alpha_{3}=1$ and $s=4$ subdomains.

The evaluation of matrix-vector products of the form $\mathbf{S}_{i} \mathbf{v}_{\Gamma}^{i}$ and $\left(\mathbf{S}_{i}\right)^{-1} \mathbf{r}_{\Gamma}^{i}$, is equivalent to solving subdomain optimal control problems (3.4) and (3.11), respectively, (cf. Theorems 3.1 and 3.2). Matrix-vector products $\mathbf{S}_{i} \mathbf{v}_{\Gamma}^{i}$ and $\left(\mathbf{S}_{i}\right)^{-1} \mathbf{r}_{\Gamma}^{i}$ are computed by solving the reduced forms of the optimal control problems (3.4) and (3.11) using the conjugate gradient method. The reduced form of the optimal control problems (3.4) or (3.11) is the one in which the state is viewed as function of the control and the optimal control problem is posed as a minimization problem in the controls only. The conjugate gradient method is stopped when the norm of the gradient is less than $10^{-10}$. The preconditioned GMRES/sQMR applied to linear operator equation (3.7) is stopped if the preconditioned residual is less than $10^{-4}$.

Table 4.1 shows that for this example, preconditioned GMRES and preconditioned sQMR behave similarly. sQMR uses a three term recurrence and therefore the amount of memory required is independent of the number of iterations, whereas in GMRES it depends linearly on the number of iterations. The number of preconditioned GMRES/SQMR iterations is roughly proportional to the square of the number of subdomains. The deterioration of the performance of the NeumannNeumann preconditioner with increasing number of subdomains is well known [5, 6, 15, 20, 21]. For elliptic PDEs and for linear-quadratic elliptic optimal control problems a coarse space is introduced to avoid this deterioration (see the references above). For linear-quadratic parabolic optimal control problems this is still under investigation. Table 4.1 also shows that the number of 
sQMR Iterations

\begin{tabular}{cc|ccc}
\multicolumn{5}{c}{$\alpha_{1}=\alpha_{2}=10, \alpha_{3}=1$} \\
$\mathrm{~s} \backslash$ & $K$ & 64 & 128 & 256 \\
\hline 2 & 1 & 1 & 1 \\
4 & & 15 & 16 & 16 \\
8 & 68 & 68 & 77 \\
16 & & 514 & &
\end{tabular}

GMRES Iterations

\begin{tabular}{rc|ccc}
\multicolumn{4}{c}{$\alpha_{1}=\alpha_{2}=10, \alpha_{3}=1$} \\
$\mathrm{~s} \backslash$ & $K$ & 64 & 128 & 256 \\
\hline 2 & 1 & 1 & 1 \\
4 & & 15 & 16 & 16 \\
8 & 54 & 55 & 60 \\
16 & & & &
\end{tabular}

SQMR Iterations

\begin{tabular}{rr|ccc}
\multicolumn{4}{c}{$\alpha_{1}=\alpha_{2}=10^{3}, \alpha_{3}=1$} \\
$\mathrm{~s} \backslash$ & $K$ & 64 & 128 & 256 \\
\hline 2 & & 1 & 1 & 1 \\
4 & 8 & 8 & 8 \\
8 & & 32 & 32 & 32 \\
16 & & 124 & 140 & 121 \\
\multicolumn{4}{c}{ GMRES Iterations }
\end{tabular}

\begin{tabular}{rc|ccc}
\multicolumn{4}{c}{$\alpha_{1}=\alpha_{2}=10^{3}, \alpha_{3}=1$} \\
$\mathrm{~s} \backslash$ & $K$ & 64 & 128 & 256 \\
\hline 2 & & 1 & 1 & 1 \\
4 & 7 & 7 & 7 \\
8 & 27 & 28 & 29 \\
16 & 95 & 99 & 103
\end{tabular}

Table 4.1: Number of preconditioned sQMR/GMRES iterations needed for the solution of (3.7) depending on the number of subdomains $s$ and on the discretization size $\Delta x=\Delta t=1 / K$.

preconditioned GMRES/sQMR iterations is insensitive to the weighting parameters $\alpha_{1}, \alpha_{2}$. The conditioning of the optimal control problem (1.1) grows as $\alpha_{1} / \alpha_{3}$ and as $\alpha_{2} / \alpha_{3}$. For larger $\alpha_{1} / \alpha_{3}$ and $\alpha_{2} / \alpha_{3}$ the problem (1.1) becomes more difficult to solve numerically. The insensitivity of the number of preconditioned GMRES iterations again matches the observations made in $[11,12]$ for Neumann-Neumann methods applied to linear-quadratic elliptic optimal control problems. The fact that preconditioned GMRES/sQMR performs better on the more difficult problem is likely due to the particular structure of our model problem, but not true in general, see, e.g., [11, 12].

\section{Conclusions}

We have presented a spatial domain decomposition (DD) preconditioner for the solution of discretized parabolic linear-quadratic optimal control problems. Our DD preconditioner is based on a decomposition of the spatial domain into non-overlapping subdomains. The optimality conditions for the parabolic linear-quadratic optimal control problem is split into smaller problems restricted to spatial subdomain-time cylinders. These subproblems correspond to parabolic linear-quadratic optimal control problems on subdomains with Dirichlet data on interfaces. The coupling of these subdomain problems leads to a Schur complement system in which the unknowns are the state and adjoint variables on the subdomain interfaces in space and time.

The Schur complement system is solved using a preconditioned GMRES or a preconditioned sQMR. The application of the Schur complement to a vector requires the (parallel) solution of smaller subdomain parabolic linear-quadratic optimal control problems. States, controls and adjoints for these smaller subdomain problems are only needed locally, but do not have to be communicated or stored globally. The preconditioner is obtained from the solution of appropriate subdomain parabolic linear-quadratic optimal control problems. The application of this preconditioner 
to a vector also requires the (parallel) solution of smaller subdomain parabolic linear-quadratic optimal control problems. Again, states, controls and adjoints for these smaller subdomain problems are only needed locally, but do not have to be communicated or stored globally.

Our numerical tests indicate that the dependence of the performance of our preconditioner on mesh size and subdomain size is similar to that of its counterpart applied to elliptic equations only and to that of its counterpart applied to elliptic linear-quadratic optimal control problems. In particular, the number of preconditioned GMRES/sQMR iterations is roughly proportional to the square of the number of subdomains. With the introduction of a coarse space one might be able to prevent this deterioration. This is subject of future research. Our tests also show that the preconditioners are insensitive to the size of the control regularization parameter.

\section{References}

[1] J.-D. Benamou. A domain decomposition method for control problems. In P. Bjørstad, M. Espedal, and D. Keyes, editors, DD9 Proceedings, pages 266-273, Bergen, Norway, 1998. Domain Decomposition Press. Available electronically from http://www.ddm.org/DD9/index.html.

[2] J. D. Benamou. Domain decomposition, optimal control of systems governed by partial differential equations, and synthesis of feedback laws. J. Optim. Theory Appl., 102(1):15-36, 1999.

[3] A. Bounaim. On the optimal control problem of the heat equation: New formulation of the problem using a non-overlapping domain decomposition technique. Technical report, Scientific Computing Group, Department of Informatics. University of Oslo, 2002.

[4] A. Comas. Time domain decomposition methods for second order linear quadratic optimal control problems. Master's thesis, Department of Computational and Applied Mathematics, Rice University, Houston, TX, 2004.

[5] M. Dryja, B. F. Smith, and O. B. Widlund. Schwarz analysis of iterative substructuring algorithms for elliptic problems in three dimensions. SIAM J. Numer. Anal., 31(3):16621694, 1994.

[6] M. Dryja and O. B. Widlund. Schwarz methods of Neumann-Neumann type for threedimensional elliptic finite element methods. Comm. Pure Appl. Math., 48:121-155, 1995.

[7] R. W. Freund and N. M. Nachtigal. A new Krylov-subspace method for symmetric indefinite linear systems. In W. F. Ames, editor, Proceedings of the 14th IMACS World Congress on Computational and Applied Mathematics, pages 1253-1256. IMACS, 1994.

[8] R. W. Freund and N. M. Nachtigal. Software for simplified Lanczos and QMR algorithms. Applied Numerical Mathematics, 19:319-341, 1995.

[9] A. Greenbaum. Iterative Methods for the Solution of Linear Systems. SIAM, Philadelphia, 1997. 
[10] M. Heinkenschloss. Time-domain decomposition iterative methods for the solution of distributed linear quadratic optimal control problems. Journal of Computational and Applied Mathematics, 173:169-198, 2005.

[11] M. Heinkenschloss and H. Nguyen. Balancing Neumann-Neumann methods for elliptic optimal control problems. In R. Kornhuber, R. H. W. Hoppe, J. Periaux, O. Pironneau, O. B. Widlund, and J. Xu, editors, Domain Decomposition methods in Science and Engineering, Lecture Notes in Computational Science and Engineering, Vol. 40, pages 589-596, Heidelberg, 2004. Springer-Verlag.

[12] M. Heinkenschloss and H. Nguyen. Neumann-Neumann domain decomposition preconditioners for linear-quadratic elliptic optimal control problems. Technical Report TR04-01, Department of Computational and Applied Mathematics, Rice University, 2004.

[13] J. E. Lagnese and G. Leugering. Time-domain decomposition of optimal control problems for the wave equation. System and Control Letters, 48:229-242, 2003.

[14] J. E. Lagnese and G. Leugering. Domain Decomposition Methods in Optimal Control of Partial Differential Equations, volume 148 of International Series of Numerical Mathematics. Birkhäuser Verlag, Basel, 2004.

[15] P. Le Tallec. Domain decomposition methods in computational mechanics. In J. T. Oden, editor, Computational Mechanics Advances, Volume 1(2), pages 121-220, New York, 1994. North Holland.

[16] J.-L. Lions. Optimal Control of Systems Governed by Partial Differential Equations. Springer Verlag, Berlin, Heidelberg, New York, 1971.

[17] Y. Maday and G. Turinici. A parareal in time procedure for the control of partial differential equations. Comptes Rendus de l'Académie des Sciences. Série I. Mathématique, 335:387392, 2002.

[18] A. Quarteroni and A. Valli. Domain Decomposition Methods for Partial Differential Equations. Oxford University Press, Oxford, 1999.

[19] Y. Saad. Iterative Methods for Sparse Linear Systems. SIAM, Philadelphia, 2nd edition, 2003.

[20] B. Smith, P. Bjørstad, and W. Gropp. Domain Decomposition. Parallel Multilevel Methods for Elliptic Partial Differential Equations. Cambridge University Press, Cambridge, 1996.

[21] A. Toselli and O. Widlund. Domain Decomposition Methods - Algorithms and Theory. Computational Mathematics, Vol. 34. Springer-Verlag, Berlin, 2004.

[22] H. A. van der Vorst. Iterative Krylov Methods for Large Linear Systems, volume 13 of Cambridge Monographs on Applied and Computational Mathematics. Cambridge University Press, Cambridge, 2003. 